\title{
Research Square \\ LEACH-MTC: A Network Energy Optimization Algorithm Constraint as Moving Target Prediction
}

\section{Zhen-tao HU}

Henan University

Yong JIN ( $\nabla_{\text {jy@henu.edu.cn ) }}$

Lin Zhou

Henan University https://orcid.org/0000-0002-2700-834X

Chen WANG

Henan University

Ji-fang LI

North China University of Water Resources and Electric Power

\section{Research}

Keywords: Wireless sensor network, state prediction, Node sleep strategy, Cluster head selection, Energy optimization

Posted Date: July 10th, 2020

DOI: https://doi.org/10.21203/rs.3.rs-39389/v1

License: (c) (1) This work is licensed under a Creative Commons Attribution 4.0 International License.

Read Full License 


\title{
LEACH-MTC : A Network Energy Optimization Algorithm Constraint as Moving Target Prediction
}

\author{
HU Zhen-tao ${ }^{1}$, JIN Yong ${ }^{1 *}$, ZHOU Lin ${ }^{1}$, WANG Chen ${ }^{1}$, LI Ji-fang ${ }^{2}$ \\ 1. College of Computer and Information Engineering Henan University, Kaifeng, 475004, China \\ 2. North China University of Water Resources and Electric Power, Zhengzhou, 450000, China. \\ * Corresponding author: jy@henu.edu.cn
}

\begin{abstract}
When some nodes cooperatively track moving targets in wireless sensor network, some things including network working node selection and network energy consumption are influenced. Thus, this paper proposes an improved algorithm LEACH-MTC (LEACH with Moving Target Constraint) based on low energy adaptive clustering hierarchy protocol (LEACH). Firstly, based on the two-step linearization of the nonlinear dynamic model, the state of nonlinear moving target is predicted by the extended Kalman filter (EKF). Secondly, combining the state prediction of moving target and the performance of collaborative monitoring, this paper constructs an ellipse monitoring area of some working nodes to consist with the direction of the target movement. Subsequently, the node sleep strategy corresponding to the state prediction of moving target is designed. Finally, the cluster head selection strategy is proposed based on energy balance utilizing the state prediction of the moving target. Simulation results show that the proposed LEACH-MTC algorithm can not only ensure the real time consistency between the changing direction of area and the direction of target movement, but also increase the number of working nodes' survival and reduce the network energy consumption.
\end{abstract}

Keywords-Wireless sensor network, state prediction, Node sleep strategy, Cluster head selection, Energy optimization

\section{INTRODUCTION}

Wireless sensor networks (WSNs) are intelligent communication networks composed of a large number of sensors in a multi-hop and self-organizing manner. WSNs have many advantages including flexible deployment, low price, low power consumption and high accuracy of information acquisition. Thus, they are widely used in military fields ${ }^{[1-3]}$, environmental monitoring ${ }^{[4-6]}$, industrial control ${ }^{[7-9]}$, and so on. However, since network nodes are usually deployed in complex, dangerous places, it is difficult to maintain them frequently, and the energy carried by the node is limited. Once the energy of nodes is exhausted, they are death, as a result, they cannot be guaranteed to detect and track targets, leads to the decline of the monitoring performance of the network subsequently. Therefore, how to effectively reduce nodes' energy consumption and prolong the lifetime of WSNs is great significance.

Sharma et.al propose LEACH-DBCH algorithm to relieve high energy consumption of classical LEACH algorithm [10]. LEACH-DBCH algorithm designs a new threshold function in cluster head selection stage. The new function comprehensively involves some factors relate to cluster head selection including remain energy of node, distance between node and base station and distance between node and cluster head. Simulations show that, comparing with classical LEACH algorithm, LEACH-DBCH algorithm can effectively prolong lifetime of WSNs. According to requirement of target monitoring quality, Guo et.al propose LEACH-RARE algorithm to save energy of WSNs by restricting the number of nodes related to target monitor and the amount of data related to cluster head ${ }^{[11]}$.

Lu proposed an efficient adaptive node selection algorithm (ANSTT) ${ }^{[12]}$, which combined the network node's perception of moving targets and the relative residual energy of network nodes to develop a working node selection mechanism. It 
effectively reduced network energy consumption and extended the network life cycle. Zhang proposed an efficient and energy-efficient adaptive network nodes scheduling algorithm ${ }^{[13]}$. By prioritizing the decision function, the algorithm used the nodes with higher value as the working nodes to balance the local energy consumption of dynamic clustering. The node with the highest value became the cluster head, and the particle filter algorithm was used to track the target whose motion model is nonlinear. Research shows that Zhang's algorithm solved the problem of unbalanced network energy consumption, which is caused by the uncertainty of target motion modeling and the randomness of cluster head selection. Moreover, Zhang's algorithm can also effectively schedule network nodes and improve the tracking accuracy of moving targets. Harizan et.al proposes a node scheduling method based on improved genetic algorithm. Fitness function of improved genetic algorithm was constructed by some factors including the least number of nodes, the selected node coverage and the remaining energy. In addition, this algorithm constructs the set of working nodes by selecting some nodes close to the target, thus leading to reduced number of nodes and enhanced network efficiency. Although the above methods can effectively reduce network resources and balance network energy consumption, some factors including network environment, static/dynamic target, and so on, which were not consider to influence working nodes selection, cluster head selection, energy consumption modeling, and so on. When the wireless sensor network nodes cooperatively track the moving target, the target motion state (for example, the motion trajectory, speed, direction, etc.) will affect the selection of the network working nodes and the network energy consumption.

In this paper, an improved algorithm named LEACH-MTC (LEACH with Moving Target Constraint) is proposed, this algorithm constructs a time-varying ellipse area in which the nodes are working nodes, these nodes format work node set and are used to monitor moving target. The proposed network node dormancy strategy considers the distance between the working node and the focuses of ellipse area. The proposed cluster head selection threshold considers the distance factor between the working node and the moving target. As a result, for the actual cooperative tracking environment, the proposed LEACH-MTC is more feasible for the realization of the moving target cooperative tracking system. Overall, the main contributions of this paper can be summarized as follows:

1) We timely construct an ellipse monitoring area with the direction changing of the moving target, which constitute a work node set to monitor the moving target. Because using two-step linearization method to linearize nonlinear dynamic system, and utilizing extended Kalman filter (EKF) to predict the moving target state, the shape and size of the ellipse area and the number of nodes contained in the area can all be changed in real time. Above can reduce the number of work nodes, thus reduce the network energy consumption caused by redundant nodes.

2) To reduce the number of working nodes, an improved network node dormancy strategy is proposed to construct working node set under the moving target tracking environment. Besides, according to the network node's own attributes and monitoring environment factors, some network monitoring accuracy indicators are reconstructed. Thus, the monitoring efficiency of system is improved.

3) Based on the working node set and the state prediction of moving target, a new cluster head selection threshold is proposed. Because the number of working nodes is considered to vary in real time, above cluster head selection strategy can be adaptively used to select the cluster head to transmit the collected data.

4) The simulations of this paper are carried out base on MATLAB platform. To better describe the feasibility and effectiveness of LEACH-MTC, we conduct designed simulation to verify the monitoring accuracy by difference working node selection strategies, the number of node survivor, the network residual energy, and so on. We can conclude that the proposed LEACH-MTC yields better performance than traditional methods. 
The rest of this paper is organized as follows. In the second section, the target nonlinear dynamic model and the target state prediction model are described. In the third section, the new network node dormancy strategy, improved cluster head selection strategy and network monitoring accuracy criterion are designed, respectively. The LEACH-MTC algorithm based on moving target constraints is proposed. In the fourth section, the feasibility and effectiveness of the improved algorithm are simulated and analyzed in terms of saving the number of working nodes and reducing the network energy consumption. The conclusions of this paper are given in the final section.

\section{SYSTEM MODEL AND CONVENTIONAL METHOD}

In the WSNs, some nodes collaborative observing target may obtain more information. We awaken some nodes in WSNs based on state prediction of target to coordinate observe target. In this section, we present target motion model, target measurement model, energy consumption model of sensor nodes and prediction mechanism of target state, respectively.

\section{A. Nonlinear motion model of target}

In a two-dimensional monitoring area, target motion model as following:

$$
\left[\begin{array}{c}
x(k+1) \\
y(k+1) \\
\theta(k+1)
\end{array}\right]=\left[\begin{array}{c}
x(k) \\
y(k) \\
\theta(k)
\end{array}\right]+\left[\begin{array}{ccc}
\Delta t & 0 & 0 \\
0 & \Delta t & 0 \\
0 & 0 & \Delta t
\end{array}\right]\left[\begin{array}{cc}
\cos \theta(k) & 0 \\
\sin \theta(k) & 0 \\
0 & 1
\end{array}\right]\left[\begin{array}{c}
\mathrm{v}(k) \\
\omega(k)
\end{array}\right]
$$

here, $x(k)$ and $y(k)$ is the coordinate of the target in $x$ and $y$ direction at time $k, \theta(k)$ is the angle between the target and the horizontal direction at time $k . \Delta t$ is the sampling time. $\mathrm{v}(k)$ is the speed of moving target, $\omega(k)$ is the angular velocity of moving target at time $k$. Assuming that the target motion is disturbed by external environmental disturbance noise, above model can be simplified as follows:

$$
\boldsymbol{X}(k+1)=f(\boldsymbol{X}(k))+\boldsymbol{W}(k)
$$

where, $\boldsymbol{X}(k)=[x(k) y(k) \theta(k)]^{\mathrm{T}}$ is the state of moving target at time $k . f(\cdot)$ is the nonlinear state transition function, which is consist in $x(k), y(k), \theta(k) . \boldsymbol{W}(k)$ is the target state Gaussian noise, and it satisfies $\boldsymbol{W}(k): N(0, \boldsymbol{Q}(k))$. $\boldsymbol{Q}(k)$ is the noise covariance matrix.

\section{B. Measurement model of target}

Assume that in the monitoring area, the deployment coordinate of the network node $s_{i}$ is $\left(x_{i}, y_{i}\right)$, and the effective communication coverage area is a circular area with the radius of the center of the network node itself as $r_{i}$. When the moving target appears within the coverage, $h_{i}(X(k))=\sqrt{\left(x(k)-x_{i}\right)^{2}+\left(y(k)-y_{i}\right)^{2}}$ can be used to express the distance between the moving target $t$ with the position $(x(k), y(k))$ and the deployed node $s_{i}$ with the position $\left(x_{i}, y_{i}\right)$. Assuming that the sensor measurement of the target is disturbed by external environmental disturbance noise, the measurement of the network node $s_{i}$ at time $k$ can be modeled as:

$$
\boldsymbol{Z}_{i}(k)=h_{i}(\boldsymbol{X}(k))+\boldsymbol{U}_{i}(k)
$$

where $\boldsymbol{X}(k)$ is the state of moving target at time $k . \boldsymbol{U}_{i}(k)$ is the measurement noise of the network node $s_{i}$ at time $k$, which is a Gaussian white noise with a mean of zero and a variance of $\boldsymbol{R}_{i}(k)$. 


\section{Two-step linearization of target state prediction}

Since the motion model of target and the measurement model of target are the equation (2) and (4), and they constitute a nonlinear detection system. They are nonlinear function, thus the standard Kalman filter (KF) is no longer applicable, and the nonlinear filtering problem needs to be approximated as a linear filtering problem, so that a suboptimal solution is obtained. Therefore, the Extended Kalman Filter (EKF) can be considered to predict or estimate the target state.

In the above nonlinear system, since the nonlinear system needs to be linearized, that is, the Taylor series expansion is used to approximate the nonlinearity, which is transformed into the process of calculating the Jacobian matrix of the nonlinear functions $f(\cdot)$ and $h(\cdot)$, that is, the state transition matrix $\boldsymbol{F}(k)$ and the observation matrix $\boldsymbol{H}(k)$ are obtained. Therefore, the linearized system model can be obtained after linearization:

$$
\left\{\begin{array}{l}
\boldsymbol{X}(k+1)=\boldsymbol{F}(k) \boldsymbol{X}(k)+\boldsymbol{W}(k) \\
\boldsymbol{Z}(k)=\boldsymbol{H}(k) \boldsymbol{X}(k)+\boldsymbol{U}(k)
\end{array}\right.
$$

where,

$$
\begin{aligned}
\boldsymbol{F}(k) & =\left[\begin{array}{ccc}
1 & 0 & -\theta(k) \\
0 & 1 & \frac{3}{2}-\theta(k) \\
0 & 0 & 1
\end{array} \|_{\theta(k)=\hat{\theta}(k \mid k-1)}\right. \\
\boldsymbol{H}(k) & =\left[\left.\begin{array}{ccc}
x(k)-x_{1} & y(k)-y_{1} & 0 \\
x(k)-x_{2} & y(k)-y_{2} & 0 \\
\mathrm{M} & \mathrm{M} & \mathrm{M} \\
x(k)-x_{n} & y(k)-y_{3} & 0
\end{array}\right|_{X(k)=\hat{x}(k \mid k-1)}\right.
\end{aligned}
$$

here, it is important to note that the equation (6) is obtained using two-step linearization. $x_{i} 、 y_{i}$ in $\boldsymbol{H}(k)$ are defined as the $\mathrm{X}$ axis and $\mathrm{Y}$-axis of $s_{i}$ nodes, respectively.

Based on the nonlinear system model (2) and (4), and the linearized system model (5), the state of moving target can be estimated or predicted by the Kalman filter. The main steps are as follows:

$$
\begin{aligned}
& \hat{\boldsymbol{X}}(k+1 \mid k)=f(\hat{\boldsymbol{X}}(k \mid k)) \\
& \boldsymbol{P}(k+1 \mid k)=\boldsymbol{F}(k) \boldsymbol{P}(k \mid k) \boldsymbol{F}^{\mathrm{T}}(k)+\boldsymbol{Q}(k) \\
& \left.\boldsymbol{K}(k+1)=\boldsymbol{P}(k+1 \mid k) \boldsymbol{H}(k) € \boldsymbol{H}(k) \boldsymbol{P}(k+1 \mid k) \boldsymbol{H}^{\mathrm{T}}(k)+\boldsymbol{R}(k)\right]^{-1} \\
& \hat{\boldsymbol{X}}(k+1 \mid k+1)=\hat{\boldsymbol{X}}(k+1 \mid k)+\boldsymbol{K}(k+1)[\boldsymbol{Z}(k+1)-h(\hat{\boldsymbol{X}}(k+1 \mid k))] \\
& \boldsymbol{P}(k+1 \mid k+1)=(\boldsymbol{I}-\boldsymbol{K}(k+1) \boldsymbol{H}(k)) \boldsymbol{P}(k+1 \mid k)
\end{aligned}
$$

D. energy consumption model of sensor nodes

We use first order model described in [15] to model energy consumption of WSNs. Considering $l$ bit data in each data packet, we describe energy consumption of node related to receiving and transmitting one data packet as follows.

$$
\begin{gathered}
E_{T X}(l, d)=E_{T X-\text { elec }}(l)+E_{T X-\text { amp }}(l, d)= \begin{cases}l E_{\text {elec }}+l \varepsilon_{f s} d^{2} & d \leq d_{0} \\
l E_{\text {elec }}+l \varepsilon_{m p} d^{4} & \text { otherwise }\end{cases} \\
E_{R X}(l)=E_{R X-\text { elec }}(l)=l E_{\text {elec }}
\end{gathered}
$$


Where $E_{T X-\text { elec }}, E_{R X \text {-elec }}$ and $E_{T X \text {-amp }}$ are defined as energy consumption on transmitter, energy consumption on receiver and energy consumption on amplifier, respectively. $\varepsilon_{f s}$ and $\varepsilon_{m p}$ are free space amplification factor and multipath attenuation amplification factor, respectively. $E_{\text {elec }}$ is energy consumption of one bit data processing. $l$ is amount of data and $d$ describe the distance between transmitter and receiver. $d_{0}=\sqrt{\varepsilon_{f s} / \varepsilon_{m p}}$ is effective communication distance between nodes.

\section{METHOD: LEACH-MTC BASED ON MOVING TARGET STATE PREDICTION}

In WSNs, when some network nodes cooperatively track a target, although the network monitoring performance is improved, the addition of a large number of working nodes will cause redundant measurement information of the target, and also cause waste of the node resources. In order to improve the utilization of nodes and reduce the energy consumption of the network, this section proposes an improved algorithm called LEACH-MTC based on the prediction information of moving targets, and it's flow chart is shown in Fig.1.

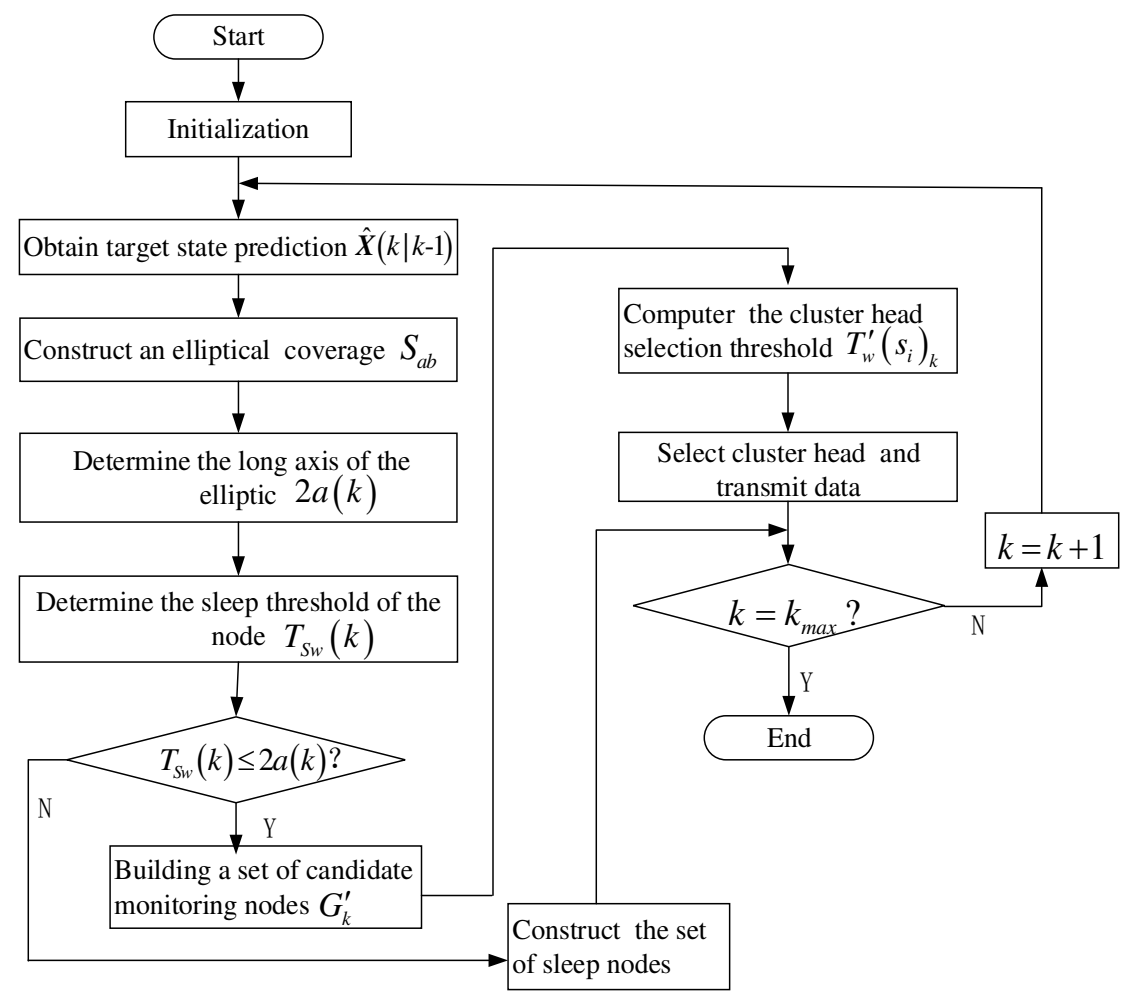

Fig.1 LEACH-MTC algorithm flow chart

Based on the LEACH protocol, the LEACH-MTC algorithm uses EKF to obtain the state prediction of moving targets in nonlinear system. In addition, an elliptical coverage area which related to the state prediction is designed to determine the working nodes. Besides, the improved node sleep strategy and the improved cluster head selection strategy are designed under the constraints of moving targets, respectively. Subsequently, three improvements including elliptic coverage construction, node sleep strategy, cluster selection strategy are introduced under some constraints of moving targets.

\section{A. Classical LEACH algorithm}

LEACH algorithm ${ }^{[16]}$ is the earliest clustering routing algorithm, which is mainly based on the process wheel and node clustering. This algorithm regards a work cycle as a round, and divides the work process into several rounds, this algorithm periodically executes the work process. Each round contains two processing: the node cluster and the data transfer. In the processing of node clustering, cluster heads of network nodes should be selected first, and a cluster head set should be 
constructed. Then, cluster heads with hierarchical structured should be formed by each cluster head node and non-cluster head nodes.

Whether some nodes become cluster heads can be judged by the threshold $T\left(s_{i}\right)$, which is as the equation (13). Assume that the value generated by node $s_{i}$ is $T_{\text {rand }}\left(s_{i}\right)$, if $T_{\text {rand }}\left(s_{i}\right) \leq T\left(s_{i}\right), s_{i}$ is selected as cluster head node, otherwise, $s_{i}$ is non-cluster head node.

$$
T\left(s_{i}\right)=\left\{\begin{array}{cc}
\frac{p}{1-p[r \bmod (1 / p)]} & s_{i} \in G \\
0 & \text { others }
\end{array}\right.
$$

here, $p$ is the ratio of the number of expected cluster head nodes selected to the total number of nodes in the monitoring area. $r$ is the current network running time (i.e. the number of running rounds). $G$ is the node set that has not been selected as cluster head in the latest round $1 / p$.

In the cluster stage, each cluster head node sends signals to the network in the form of broadcast. According to the received signal strength, each non-cluster head node chooses the cluster head to join. In order to reduce network energy consumption, non-cluster head nodes are usually added to the nearest cluster head. When all the non-cluster head nodes are added to the corresponding cluster head, all the nodes complete the cluster forming.

In the data transmission stage, cluster member nodes send some monitoring data to the cluster head according to the timing schedule. Then the cluster head fuses the received data and transmits the fusion results to the base station.

\section{B. Construction of elliptical area based on state prediction}

Intuitively, reasonably choosing work nodes close to target can effectively reduce redundant information related to observed target, thus enhancing energy efficiency of WSNs. So, we can pre-awaken sleeping nodes nearby direction of moving target to perform monitor work. Specifically, to make sure working nodes (i.e. awakened sleeping nodes) located in direction of moving target, coverage area of awakened sleeping nodes is defined as an ellipse whose major axis coincides with the direction of target moving.

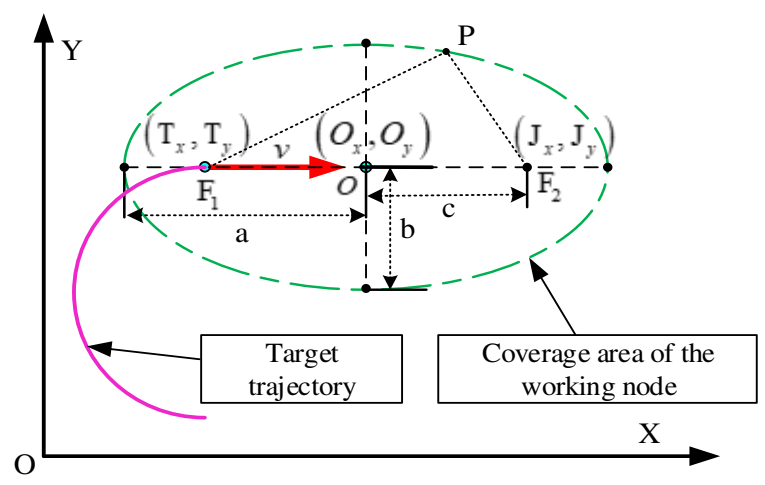

Fig. 2 Target motion trajectory and elliptical area

According to the network monitoring performance parameter $C_{w}$ and the target state prediction, an elliptical coverage area can be constructed. The size of the coverage area can be represented by an elliptical area $S_{a b}$ as follows:

$$
S_{a b}=\pi a b=\frac{C_{w} S_{M}}{m}
$$


where $a$ and $b$ represent the long semi-axis, the short semi-axis of the elliptical coverage area, respectively. $C_{w}$ represents the monitoring performance of network. $S_{M}$ represents the monitored area, and $m$ represents the total number of nodes deployed in the network. The schematic figure based on the motion trajectory of moving target and the elliptical coverage area of the working node is shown in Fig.2.

In Fig.2, the nodes inside the ellipse are in the working state, while the nodes outside the ellipse are in the sleep state. $O\left(O_{x}, O_{y}\right)$ is the coordinate of the elliptical center. $\mathrm{F}_{1}\left(\mathrm{~T}_{x}, \mathrm{~T}_{y}\right)$ is the back focus of the ellipse, and it represents the current position of the moving target. The long axis of elliptical is in the direction of the target motion, and $v$ represents the current speed of the moving target, and its direction is the tangential direction of the target moving trajectory. $\mathrm{F}_{2}\left(\mathrm{~J}_{x}, \mathrm{~J}_{y}\right)$ is the front focus of the ellipse and it represents the prediction position of the moving target. $a$ is the long semi-axis of the ellipse, $b$ is the short semi-axis of the ellipse, $c$ is the semi-focal length of the ellipse. If $\mathrm{P}$ is any point on the elliptic curve, it satisfies the sum of the distances to the two focal points equal to the long axis, which is equation (15), otherwise, if $\mathrm{P}$ is any point inside the ellipse, it has the equation (16):

$$
\begin{aligned}
& \left|\mathrm{PF}_{1}\right|+\left|\mathrm{PF}_{2}\right|=2 a \\
& \left|\mathrm{PF}_{1}\right|+\left|\mathrm{PF}_{2}\right|<2 a
\end{aligned}
$$

According to the equations (15) and (16), this paper determines whether the network node in the working state or in the sleep state. The distribution of network nodes as shown in Fig. 3.

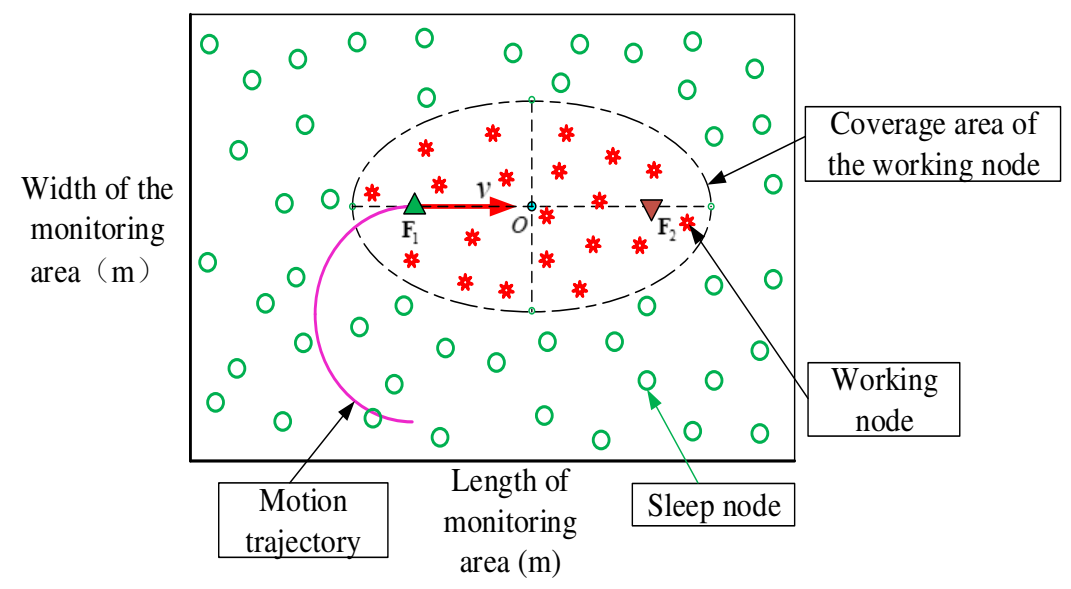

Fig. 3 Distribution of working nodes and sleep nodes

In Fig.3, $\bigcirc$ represents a network node in a sleep state, and * represents a network node in a working state. The elliptical area is the area of the coverage area formed by the working node, where $F_{1}$ is the current position of the moving target and $\mathrm{F}_{2}$ is another focus about the center $O$ of ellipse.

Based on the Fig.2, at time $k$, we assume that the coordinate of the elliptical center is $O\left(O_{x}(k), O_{y}(k)\right)$, the two focal coordinates of the ellipse are $\mathrm{F}_{1}\left(\mathrm{~T}_{x}(k), \mathrm{T}_{y}(k)\right)$ and $\mathrm{F}_{2}\left(\mathrm{~J}_{x}(k), \mathrm{J}_{y}(k)\right)$, respectively. According to the state prediction of the moving target, the predicted position coordinate can be received as $\left(\mathrm{T}_{x}\left(k+\delta_{t}\right), \mathrm{T}_{y}\left(k+\delta_{t}\right)\right)$ at time $k+\delta_{t}$. Thus, the magnitude of speed $v(k)$ as follows:

$$
|v(k)|=\frac{\sqrt{\left(\mathrm{T}_{x}\left(k+\delta_{t}\right)-\mathrm{T}_{x}(k)\right)^{2}+\left(\mathrm{T}_{y}\left(k+\delta_{t}\right)-\mathrm{T}_{y}(k)\right)^{2}}}{\delta_{t}}
$$


We assume that the shape of the elliptical coverage area formed is related to the target motion velocity, then the semifocal length of the ellipse $c(k)$ is received by the equation (18)

$$
c(k)=\frac{1}{2}\left|\mathrm{~F}_{1} \mathrm{~F}_{2}\right|=\lambda|v(k)|
$$

where $\lambda$ is the weight coefficient. According to the relation between the axis and the focal length of the ellipse, there is a relation is $a(k)^{2}=b(k)^{2}+c(k)^{2}$. Combining the elliptical coverage area $S_{a b}(k)$ in the equation (14), the ellipse long semiaxis $a(k)$ and the short semi-axis $b(k)$ are obtained as follows, respectively:

$$
\begin{aligned}
& a(k)=\sqrt{2} S_{a b}(k) /\left(\pi \sqrt{\frac{\sqrt{(\lambda|v(k)|)^{4} \pi^{2}+4 S_{a b}(k)}}{\pi}}-(\lambda|v(k)|)^{2}\right) \\
& b(k)=\frac{\sqrt{2}}{2} \sqrt{\frac{\sqrt{(\lambda|v(k)|)^{4} \pi^{2}+4 S_{a b}(k)}}{\pi}-(\lambda|v(k)|)^{2}}
\end{aligned}
$$

Thus, based on the moving target's current position $\mathrm{F}_{1}$ and the velocity $v(k)$, the position of the front focus $\mathrm{F}_{2}\left(\mathbf{J}_{x}(k), \mathbf{J}_{y}(k)\right)$ and the center $O\left(O_{x}(k), O_{y}(k)\right)$ of the ellipse can be obtained:

$$
\begin{aligned}
& {\left[\begin{array}{l}
O_{x}(k) \\
O_{y}(k)
\end{array}\right]=\left[\begin{array}{c}
\mathrm{T}_{x}(k) \\
\mathrm{T}_{y}(k)
\end{array}\right]+\lambda \mid v(k)\left[\begin{array}{ll}
1 & 0 \\
0 & 1
\end{array}\right]\left[\begin{array}{c}
\cos \theta(k) \\
\sin \theta(p)
\end{array}\right]} \\
& {\left[\begin{array}{l}
\mathrm{J}_{x}(k) \\
\mathrm{J}_{y}(k)
\end{array}\right]=\left[\begin{array}{l}
O_{x}(k) \\
O_{y}(k)
\end{array}\right]+\lambda \mid v(k)\left[\begin{array}{ll}
1 & 0 \\
0 & 1
\end{array}\right]\left[\begin{array}{l}
\cos \theta(k) \\
\sin \theta(k)
\end{array}\right]}
\end{aligned}
$$

where $\mathscr{\theta}(k)$ is the predicted of $\theta(k)$ which is the angle between the target and the horizontal direction at time $k$.

In summary, the shape and size of the elliptical coverage area constructed can be determined by the ellipse area $S_{a b}(k)$, the long semi-axis $a(k)$, the short semi-axis $b(k)$, the focal point $\mathrm{F}_{2}$, and the ellipse center $O(k)$. According coverage area of ellipse, nodes in ellipse should be awakened and nodes out of ellipse should be kept in sleeping.

\section{Node sleep strategy under moving target constraint}

In ellipse, based on the back focus $\mathrm{F}_{1}\left(\mathrm{~T}_{x}, \mathrm{~T}_{y}\right)$ and the front focus $\mathrm{F}_{2}\left(\mathrm{~J}_{x}, \mathrm{~J}_{y}\right)$, the sleep threshold $T_{S w}(k)$ of the network node $s_{i}\left(x_{i}, y_{i}\right)$ can be defined at time $k$, and it is as follows:

$$
T_{S w}(k)=\sqrt{\left(x_{i}-\mathrm{T}_{x}(k)\right)^{2}+\left(y_{i}-\mathrm{T}_{y}(k)\right)^{2}}+\sqrt{\left(x_{i}-\mathrm{J}_{x}(k)\right)^{2}+\left(y_{i}-\mathrm{J}_{x}(k)\right)^{2}}
$$

Therefore, a strategy of sleep node is developed, which can select working nodes more effectively and cost-effectively, and reduce the number of working nodes and network energy consumption:

a) If $T_{S w}(k) \leq 2 a(k)$, the node $s_{i}$ inside the elliptical coverage area, it is in working state and performs the monitoring task, at the same time, this node $s_{i}$ is added to the working node set $G_{k}^{\prime}$;

b) If $T_{S w}(k)>2 a(k)$, the node $s_{i}$ is not in the elliptical coverage area, it is in a sleep state and does not perform any monitoring task. 


\section{Cluster head selection strategy based on equilibrium}

In this section, round is defined as base unit related to data transmission in network. In each round, after the working node set $G_{k}^{\prime}$ is formed, cluster head selection is performed for all working nodes. In the cluster head selection stage, in order to reduce the probability that nodes with little residual energy or far from the base station become cluster heads, an improved strategy is designed. This strategy takes into some factors including target movement state, the distance between working node and the target, the residual energy rate of working node, and the distance between working node and the base station. Assuming $s_{i}$ is a working node, we can obtain it's the cluster head selection threshold $T_{w}^{\prime}\left(s_{i}\right)_{k}$ at time $k$ :

$$
\begin{aligned}
& T_{w}^{\prime}\left(s_{i}\right)_{k}=\left\{\begin{array}{cc}
\kappa * p_{k}+\mu * \Delta p_{k} & s_{i} \in G_{k}^{\prime} \\
0 & \text { others }
\end{array}\right. \\
& \Delta p_{k}=\Delta T\left(s_{i}\right)_{k}-\frac{1}{n_{k}} \sum_{i=1}^{n_{k}} \Delta T\left(s_{i}\right)_{k} \\
& \Delta T\left(s_{i}\right)_{k}=\alpha E_{\text {arg }}\left(s_{i}\right)_{k}+\beta\left(1-\omega_{s t}\left(s_{i}\right)_{k}\right)+\gamma\left(1-\omega_{s s}\left(s_{i}\right)_{k}\right)
\end{aligned}
$$

where $p_{k}$ and $\Delta p_{k}$ represent the expected probability, the compensation probability which the node $s_{i}$ becomes the cluster head, respectively. $n_{k}$ represents the number of surviving nodes in the working node set $G_{k}^{\prime}$. $E_{\text {arg }}\left(s_{i}\right)_{k}$ represents the residual energy rate of the node $\mathrm{s}_{i} . \omega_{s t}\left(\mathrm{~s}_{i}\right)_{k}$ and $\omega_{s s}\left(s_{i}\right)_{k}$ represent the distance factor of the node $\mathrm{s}_{i}$ to the target, the node $\mathrm{s}_{i}$ to the base station, respectively. $\kappa, \mu, \alpha, \beta$ and $\gamma$ represent the weighting coefficient of the cluster head selection threshold, respectively.

Therefore, after the cluster head selection threshold is determined using the equation (24), the cluster can be formed by the LEACH protocol framework. Every cluster with working nodes which is self-organized, and the monitoring data of the nodes are processed and transmitted by the cluster head nodes.

\section{E. Network monitoring accuracy}

It is assumed that the position of the moving target is $\mathrm{T}\left(x_{\mathrm{T}}(k), y_{\mathrm{T}}(k)\right)$ at time $k$, every working node $s_{i}$ inside the elliptical area has its own measurement $Z_{s_{i}}\left(x_{\mathrm{T} i}(k), y_{\mathrm{Ti}}(k)\right)$ for the moving target. Since the node measurements are subject to environmental interference during the monitoring process, and as the distance $d_{\mathrm{T} i}(k)$ between the working node and the moving target increases, the interference effect becomes more serious. Therefore, the measurement of working node $s_{i}$ can be expressed as follows:

$$
\begin{aligned}
& Z_{s_{i}}\left(x_{\mathrm{T} i}(k), y_{\mathrm{T} i}(k)\right)=T\left(x_{\mathrm{T}}(k), y_{\mathrm{T}}(k)\right)+f\left(\varepsilon, d_{\mathrm{Ti}}(k)\right)+\boldsymbol{V}(k) \\
& d_{\mathrm{Ti}}(k)=\sqrt{\left(x_{i}-x_{\mathrm{T}}(k)\right)^{2}+\left(y_{i}-y_{\mathrm{T}}(k)\right)^{2}}
\end{aligned}
$$

where the position of the working node $s_{i}$ is $\left(x_{i}, y_{i}\right)$ which is fixed deployment. $f(\cdot)$ is a function of the working node monitoring accuracy and distance, here, $\varepsilon$ is a weighting factor. $\boldsymbol{V}(k)$ is a Gaussian white noise.

In order to ensure high monitoring accuracy, it is necessary to enable multiple nodes to work simultaneously. Thus, more state information of the moving target can be obtained. But, in order to select the appropriate number of working nodes to reduce network resource, the network monitoring accuracy can be developed by the difference between the actual and the 
expected value of the selected working node. Besides, $\Delta Z_{\mathrm{T}}(k)$ represents the measurement error of the working node, and it is as follows:

$$
\Delta Z_{\mathrm{T}}(k)=\frac{\left\|\frac{1}{m} \sum_{i=1}^{m} Z_{s_{i}}\left(x_{\mathrm{T} i}(k), y_{\mathrm{T} i}(k)\right)-\mathrm{T}\left(x_{\mathrm{T}}(k), y_{\mathrm{T}}(k)\right)\right\|_{2}}{\left\|\mathrm{~T}\left(x_{\mathrm{T}}(k), y_{\mathrm{T}}(k)\right)\right\|_{2}} \times 100 \%
$$

where $m$ represents the number of working nodes at time $k$.

According to the size of the network monitoring area and the monitoring environment, it can be set that the network monitoring accuracy error can meet the monitoring accuracy requirements as long as it satisfies $\Delta Z_{\mathrm{T}}(k) \leq \Phi \%$, here, $\Phi \%$ is the threshold of accuracy.

\section{SimUlation RESUlTS AND DISCUSSION}

\section{A. Simulation background and parameters}

When multiple nodes monitor the moving target, some network nodes in the vicinity of target are selected to perform monitoring tasks. These working nodes need to consume energy for tasks such as information collection, data processing, and communication transmission. Once the remaining energy of one working node is less than zero, it is considered dead and loses itself monitoring ability. Network nodes that are inactive can be considered dormant and they do not consume their own energy.

In this section, performances of proposed method are verified by comparative experiments on MATLAB simulation platform. The main parameters of simulation are shown in Table I. Besides, the proposed algorithm LEACH-MTC is compared to three other LEACH-based algorithms: LEACH ${ }^{[16]}, \mathrm{LEACH} \mathrm{DBCH}^{[10]}$ and LEACH-RARE ${ }^{[11]}$, and conducts performance analysis from the aspects of the working nodes selection, nodes survival number, network residual energy and network monitoring accuracy, and so on.

TABLE I. Simulation parameters

\begin{tabular}{l|l|l}
\hline \multicolumn{1}{c|}{ Parameter name } & \multicolumn{1}{c}{ Symbol } & \multicolumn{1}{c}{ Value } \\
\hline Network monitoring area & $S_{M}$ & $300 \mathrm{~m} \times 300 \mathrm{~m}$ \\
\hline Number of network nodes & $m$ & 100 \\
\hline Base station location & $\left(x_{B S}, y_{B S}\right)$ & $(150,150)$ \\
\hline Initial energy of the node & $E_{0}$ & $1 \mathrm{~J}$ \\
\hline Transmitting circuit energy consumption & $E_{T X} / E_{R X}$ & $50 \mathrm{~nJ} / \mathrm{bit}$ \\
\hline $\begin{array}{l}\text { Signal amplification energy consumption in unit data } \\
\text { free space }\end{array}$ & $\varepsilon_{f s}$ & $10 \mathrm{pJ} / \mathrm{bit} / \mathrm{m}^{2}$ \\
\hline $\begin{array}{l}\text { Signal amplification energy consumption under unit } \\
\text { data multipath attenuation }\end{array}$ & $\varepsilon_{m p}$ & $0.0013 \mathrm{pJ} / \mathrm{bit} / \mathrm{m}^{2}$ \\
\hline Data fusion energy consumption & $\mathrm{E}_{\mathrm{DA}}$ & $5 \mathrm{~nJ} / \mathrm{bit} / \mathrm{packet}$ \\
\hline Control signal size & $l_{1}$ & $100 \mathrm{bit}$ \\
\hline Packet size & $l_{2}$ & $4000 \mathrm{bit}$ \\
\hline Expected probability of cluster head & $p_{e x}$ & 0.1 \\
\hline Maximum number of running rounds & $k_{\max }$ & 1300 \\
\hline Threshold weight & $\kappa$ & 0.8 \\
\hline Threshold weight & $\mu$ & 0.2 \\
\hline Threshold weight & $\alpha, \beta, \gamma$ & $10,0.01,0.01$ \\
\hline
\end{tabular}




\section{B. Verification of proposed algorithm}

In the comparative simulation, the distribution of network nodes and working nodes corresponding to the four algorithms of LEACH, LEACH-DBCH, LEACH-RARE and LEACH-MTC is shown in Fig. 4.

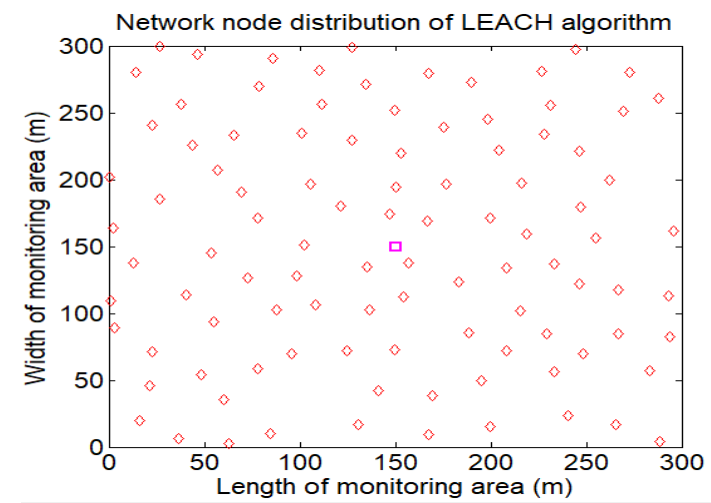

(a)

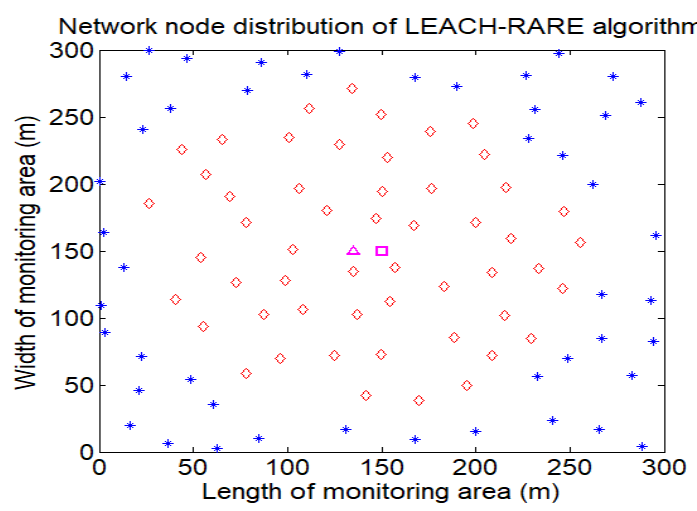

(c)

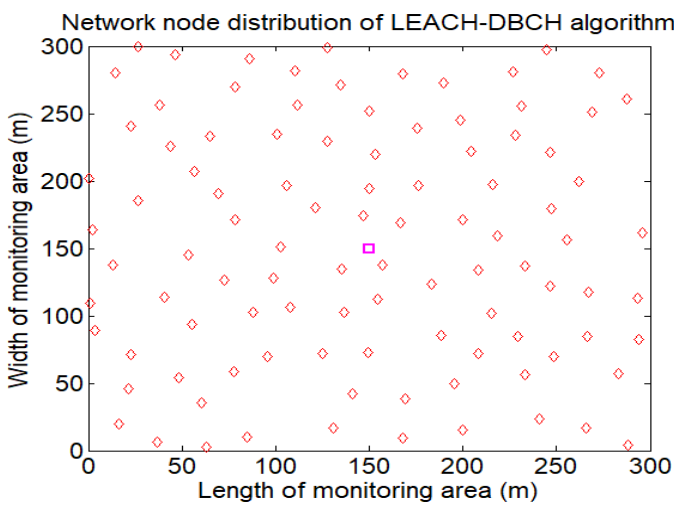

(b)

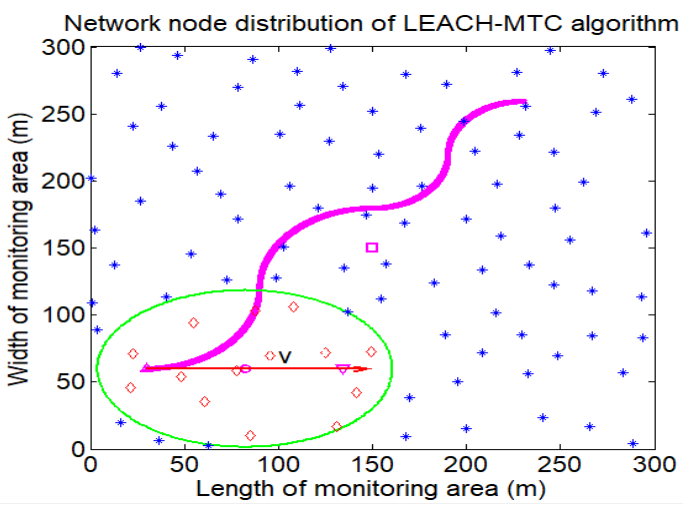

(d)

Fig.4 Node distribution of four algorithms

In Fig.4, $\square$ stands for base station, $\Delta$ stands for moving target, $\diamond$ stands for working node, $*$ stands for sleep node, curve stands for target trajectory, the target velocity at the current moment is $v$, and the direction is horizontal. In (a) and (b), the network nodes in the monitoring area corresponding to the LEACH and LEACH-DBCH algorithms are all in working state. Since the two algorithms do not consider the factor of the moving target, all nodes in the network are working nodes. They all need to collect data, process data and transmit data. But, in (c), the nodes closer to the target in the LEACH-RARE algorithm are in the working state, while the nodes farther from the target are in the dormant state, and there are 52 working nodes and 48 sleep nodes. In (d), the nodes inside the elliptical area are in working state, and their number is 14 , the nodes outside the elliptical area are in the dormant state, and as the position of the moving target changes, the shape and size of the ellipse area also changes.

\section{a) Working node selection and monitoring accuracy}

As the target moves, the network nodes that are working are constantly changing. Fig.5 shows the change of the target position and the working node when the LEACH-MTC algorithm is running in the 1th round, the 300th round, the 600th round and the 800th round, respectively.

In Fig.5, $\square$ stands for base station, $\Delta$ stands for target, $\diamond$ stands for working node, $*$ stands for sleep node, curve stands for target trajectory. In (a) and (c), when the number of network running rounds is in the 1th round and the 600th round, the number of nodes that are in the working state is 15 , and the remaining nodes are in a dormant state. In (b) and (d), when the 
number of network running rounds is 300th round and 800th round, the number of nodes that are in working state is 16 . Above results indicate that as the target position changes, the long axis, the short axis and the shape of the ellipse also change, besides, the number of working nodes that are in the ellipse area is constantly change.

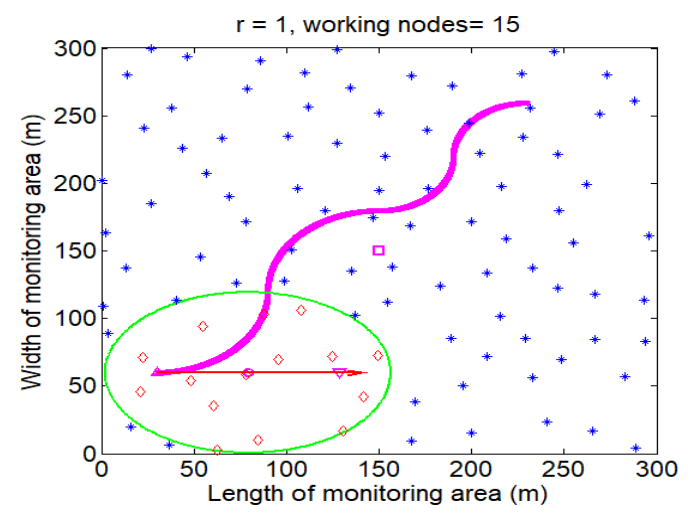

(a)

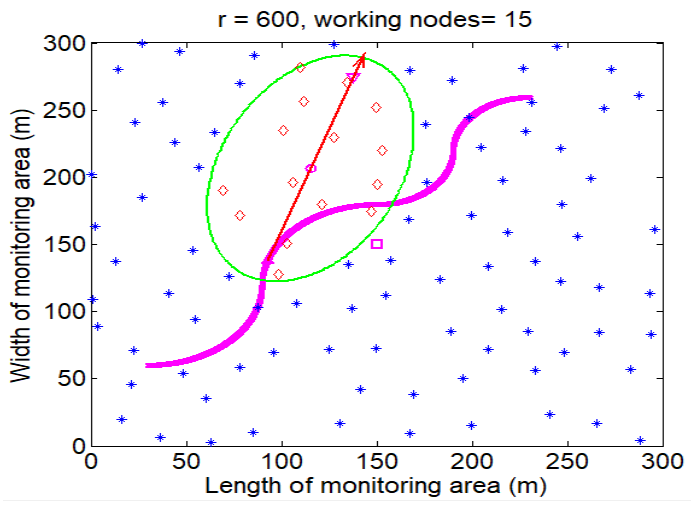

(c)

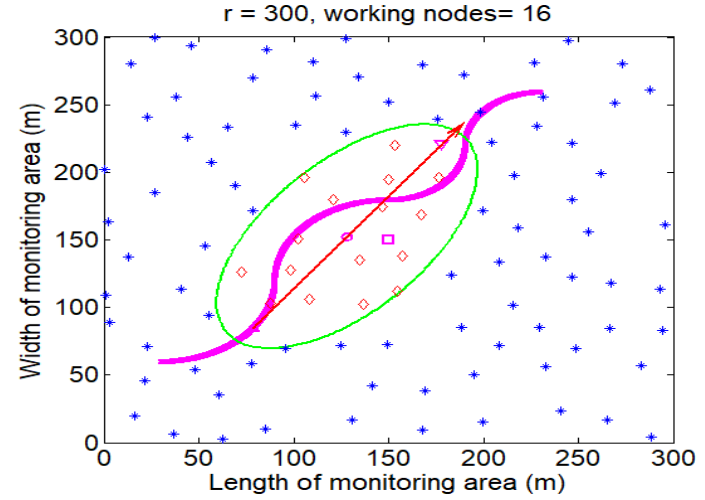

(b)

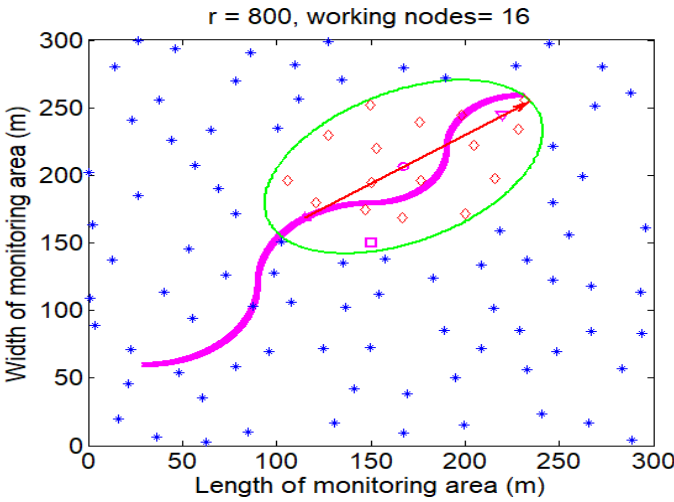

(d)

Fig.5 Change of target position and working node selection

Assume that the elliptical area are formed by the working node is $S_{a b}=14400 \mathrm{~m}^{2}$, and all the working nodes in this area can meet the monitoring accuracy requirements. According to equations (17), (18), (19) and (20), the long semi-axis $a$, the short semi-axis $b$ and the half-focal length $c$ of the ellipse area can be obtained. The network monitoring accuracy is shown in Table II:

TABLE II. Ellipse parameters and network monitoring error

\begin{tabular}{l|c|c|c|c}
\hline \multicolumn{1}{c|}{ Run time parameter } & Round 1 & Round 300 & Round 600 & Round 800 \\
\hline Number of working nodes & 15 & 16 & 15 & 16 \\
\hline long semi-axes $a$ & $79.1984 \mathrm{~m}$ & $96.6545 \mathrm{~m}$ & $86.9920 \mathrm{~m}$ & $82.9734 \mathrm{~m}$ \\
\hline short semi-axes $b$ & $57.8757 \mathrm{~m}$ & $47.4232 \mathrm{~m}$ & $49.3975 \mathrm{~m}$ & $51.7899 \mathrm{~m}$ \\
\hline Half focal length $c$ & $54.0629 \mathrm{~m}$ & $84.2208 \mathrm{~m}$ & $71.6066 \mathrm{~m}$ & $64.8259 \mathrm{~m}$ \\
\hline Network monitoring error & $1.85 \%$ & $2.10 \%$ & $1.73 \%$ & $1.65 \%$ \\
\hline
\end{tabular}

From Table II, it can be seen that the number of running rounds is the 1 th round, the 300 th round, the 600th round, and the 800th round, respectively, the number of working nodes in the elliptical area is not the same, which indicates that as the target position changes, the ellipse area also changes. It can be seen from the changes of the long semi-axes, the short semiaxes and the semi-focal lengths in Table II. This indicates that as the target position changes constantly, the shape of the ellipse area also changes. The analysis of the monitoring accuracy of the network at four moments shows that the monitoring error is less than $5 \%$, which can meet the requirements of network monitoring accuracy.

b) Number of node survivor and network residual energy 


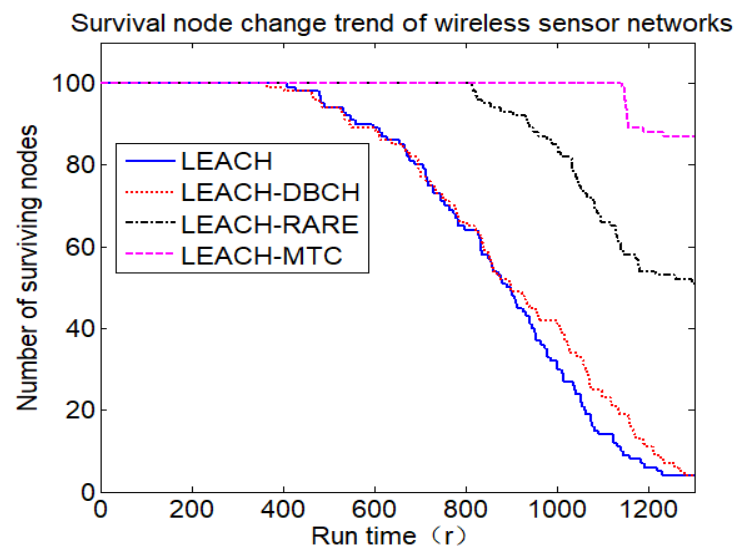

Fig. 6 The number of network surviving nodes

The working nodes need to constantly obtain the moving target state, and as the amount of data processing increases gradually, the energy consumption of the working nodes increases continuously, their residual energy decreases gradually. When the residual energy of the working node is less than 0 , it is regarded as the dead state, which leads to the reduction of the number of surviving nodes. Length of time from network start running to first nodes death is defined as the life cycle of the network. Fig. 6 shows the changes of the number of the surviving nodes corresponding to the four algorithms. In Fig.6, when the network starts running, there are 100 surviving nodes corresponding to the four algorithms. As the network running time is extended, the energy consumption of the working nodes will become larger and larger, and the remaining energy of the working nodes will gradually decrease. It can be seen from this figure that when the run-time corresponding to the four algorithms run to $407,365,814$ and 1142 , respectively, the four corresponding curves begin to decline, that is, the death nodes begin to appear in the network. By comparing the time of death of the first node of the four algorithms, it can be seen that the LEACH-MTC algorithm has the longest time to appear the first dead node. Therefore, the LEACH-MTC algorithm can effectively extend the time of the first dead node. When the number of running rounds is the same, the curve corresponding to the LEACH-MTC algorithm is above the curve corresponding to the other three algorithms in the whole process, which indicates that the LEACH-MTC algorithm has the largest number of network surviving nodes when the running time is the same. When the network runs to the maximum number of running rounds i.e. $k_{\max }=1300$, the number of surviving nodes in the network corresponding to the four algorithms is $4,4,51$, and 87 , respectively. In summary, the LEACH-MTC algorithm makes the number of surviving nodes higher than that of other three algorithms; it effectively increases the number of nodes surviving. Accordingly, Fig.7 shows the variation trend of the total residual energy of network nodes with the working time.

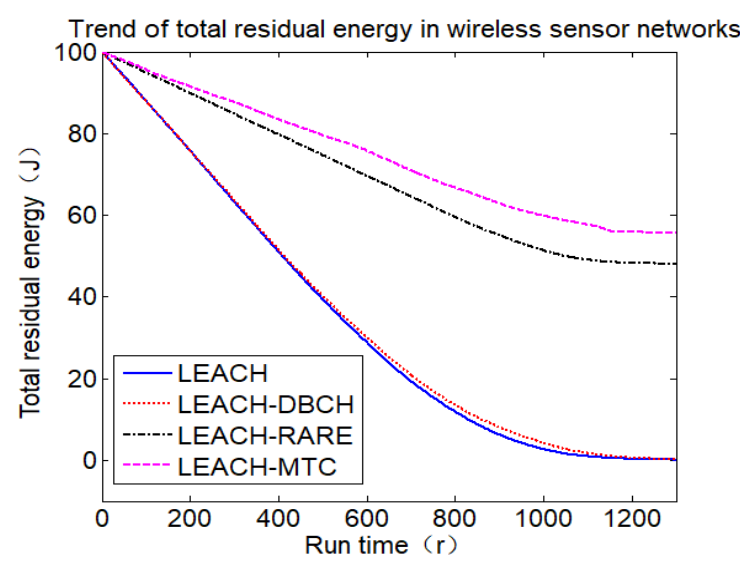

Fig.7 The variation of network residual energy 
In Fig.7, the total initial energy of the four algorithms is 100J. As the network running time increases, the energy curves corresponding to the four algorithms all have a downward trend. When the number of running rounds is the same, the curve of the LEACH-MTC algorithm is obviously above the other three curves, which means that the network residual energy of the algorithm is larger than the other three algorithms. When the network runs to the maximum running rounds i.e. $k_{\max }=1300$, the remaining energy of the network nodes of the algorithms LEACH, LEACH-DBCH, LEACH-RARE and LEACH-MTC are $0.0807 \mathrm{~J}, 0.2345 \mathrm{~J}, 48.0405 \mathrm{~J}$ and $55.6336 \mathrm{~J}$, respectively. It can be seen that the total residual energy of the LEACH-MTC algorithm is higher than that of the other three algorithms. The simulation results show that the residual network energy corresponding to the four algorithms decreases with the increase of running time, but the residual network energy corresponding to LEACH-MTC algorithm is higher than the other three algorithms, which can save network energy more effectively and prolong network life cycle.

\section{CONCLUSION}

The proposed LEACH-MTC algorithm combines the state prediction of moving target and the network monitoring performance parameters, it meets the network monitoring accuracy requirements. This paper constructs a time-varying ellipse monitoring area with the direction changing of the moving target, and it determines the working nodes set. Combined the working node set and the state prediction of moving target, this paper gives a new cluster head selection threshold to construct the cluster head strategy. The LEACH-MTC algorithm increases the number of network nodes to survive, reduces network energy consumption, and prolongs the network life time.

\footnotetext{
Abbreviations

LEACH: Low energy adaptive clustering hierarchy protocol; LEACH-MTC: LEACH with Moving Target Constraint;

EKF: Extended Kalman filter;

WSNs: Wireless sensor networks;

ANSTT: Adaptive node selection algorithm;

KF: Kalman filter;
}

\section{Acknowledgment}

The authors would like to thank the anonymous referees for their helpful suggestions.

\section{Authors' contributions}

HU conceived and designed the study. JIN and ZHOU construct and perform experiments and simulation. HU wrote this paper. LI revised this paper. A11 authors read and approved the manuscript.

\section{Authors' information}

Zhentao HU received the M. S. in application mathematics from Henan University, Kaifeng, China, in 2006, and a Ph. D degree in control science and engineering from Northwestern Polytechnical University China, in 2010. Now, he is an associate professor of college of computer and information engineering, Henan University. His research interests include target tracking, nonlinear estimation and particle filtering.

Yong JIN received B.S. in Electrical Engineering from Tongji University, Shanghai, China, in 1994 and Ph. D. degrees in information and communication engineering from Northwestern Polytechnical University, Xi' an, China, in 2010. Since 2015, he was as a professor with the college of computer and information engineering. Also, he has served as a peer-reviewer for various IEEE research journals since 2010. His research interests include array signal processing and statistical signal processing.

Lin ZHOU received the M.S. in application mathematics from Henan University, Kaifeng, China, in 2005, and the Ph. D degree in control theory and control engineering from Northwestern Polytechnical University China, in 2013. Now, she is an associate professor of college of computer and information engineering, Henan University. Her research interests include information fusion and sensor management.

Chen WANG received the B.S. degree in automation from the Henan University, Kaifeng, China, in 2018, where he is current1y working toward the M.S. degree. He is currently a student member of the lab of intelligent technology and systems of Henan University and his research interests include wireless sensor networks and target tracking.

Jifang LI received the M. S. in control engineering from Huazhong University of Science and Technology, Zhengzhou, China, in 2003, and a Ph. D degree in power electronics and power drives from Shanghai Maritime University China, in 2011. Now, he is a professor of electric power college, North China University of Water Resources and Electric Power. His research interests include power electronics and power drives, new energy power generation and smart grid. 


\section{Funding}

This work was supported by the National Science Foundation Council of China (61771006, 61976080, U1804149), Key research projects of university in Henan province of china (19A413006, 20B510001), First-class Discipline Training Foundation of Henan University (2018YLTD04), the Programs for Science and Technology Development of Henan Province (192102210254).

\section{Availability of data and materials}

Because all data have been obtained by the simulation model and MATLAB source code, our data sharing is unnecessary.

\section{Competing interests}

A11 authors declare that they have no competing interests.

\section{REFERENCES}

[1] Sundararaj V, Muthukumar S, Kumar R S, et al. An optimal cluster formation based energy efficient dynamic scheduling hybrid MAC protocol for heavy traffic load in wireless sensor networks[J]. Computers \& Security, 2018: $277-288$.

[2] Sheng Z, Mahapatra C, Leung V C, et al. Energy Efficient Cooperative Computing in Mobile Wireless Sensor Networks[C]. Ieee International Conference on Cloud Computing Technology and Science, 2018, 6(1): 114-126.

[3] Sarkar A, Murugan T S. Cluster head selection for energy efficient and delay-less routing in wireless sensor network[J]. Wireless Networks, 2019, 25(1): 303-320.

[4] Mostafaei H. Energy-Efficient Algorithm for Reliable Routing of Wireless Sensor Networks[J]. IEEE Transactions on Industrial Electronics, 2019, 66(7): 5567-5575.

[5] Guo S, Shi Y, Yang Y, et al. Energy Efficiency Maximization in Mobile Wireless Energy Harvesting Sensor Networks[J]. IEEE Transactions on Mobile Computing, 2018, 17(7): 1524-1537.

[6] Mehmood A, Lv Z, Lloret J, et al. ELDC: An Artificial Neural Network Based Energy-Efficient and Robust Routing Scheme for Pollution Monitoring in WSNs[J]. IEEE Transactions on Emerging Topics in Computing,2020,8(1): 106114.

[7] Lu W, Gong Y, Liu X, et al. Collaborative Energy and Information Transfer in Green Wireless Sensor Networks for Smart Cities[J]. IEEE Transactions on Industrial Informatics, 2018, 14(4): 1585-1593.

[8] Zhan C, Zeng Y, Zhang R. Energy-efficient data collection in UAV enabled wireless sensor network[J]. IEEE Wireless Communications Letters, 2017, 7(3): 328-331.

[9] Mekikis P V, Kartsakli E, Antonopoulos A, et al. Connectivity analysis in clustered wireless sensor networks powered by solar energy[J]. IEEE Transactions on Wireless Communications, 2018, 17(4): 2389-2401.

[10] R Sharma, N Mishra, S Srivastava. A proposed energy efficient distance based cluster head (DBCH) algorithm: An improvement over LEACH [J]. Procedia Computer Science, 2015, 57(7): 807-814.

[11] M Guo, E Olule, G Wang, et al. Designing energy efficient target tracking protocol with quality monitoring in wireless sensor networks [J]. Journal of Supercomputing, 2010, 51(2): 131-148.

[12] X Lu, L Cheng, S L Luo. Adaptive node scheduling algorithm for target tracking in wireless sensor networks [J]. Journal on Communications, 2015, 36(4):70-80.

[13] J Zhang. Energy-efficient adaptive dynamic sensor scheduling for target monitoring in wireless sensor networks[J]. ETRI Journal, 2011, 33(6): 857-863.

[14] Harizan S, Kuila P. Coverage and connectivity aware energy efficient scheduling in target based wireless sensor networks: an improved genetic algorithm based approach[J]. Wireless Networks, 2019, 25(4): 1995-2011.

[15] Kang J, Sohn I, Lee S H. Enhanced message-passing based LEACH protocol for wireless sensor networks[J]. Sensors, 2019, 19(1): 75-91.

[16] W Heinzelman, A Chandrakasan, H Balakrishnan. An application-specific protocol architecture for wireless microsensor networks [J]. IEEE Transactions on wireless communications, 2002, 1(4): 660-670. 


\section{Description of figure}

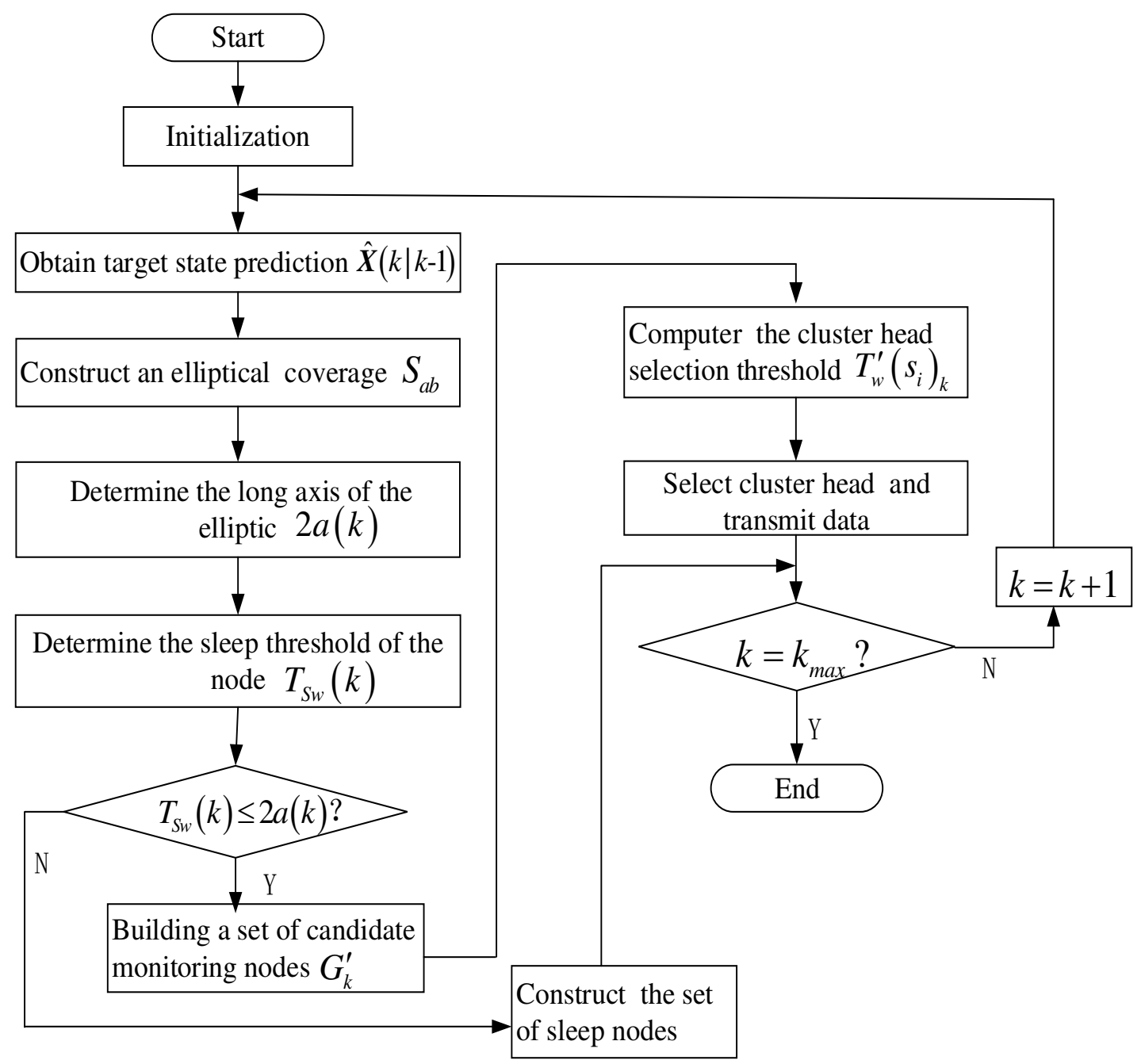

Fig.1 LEACH-MTC algorithm flow chart

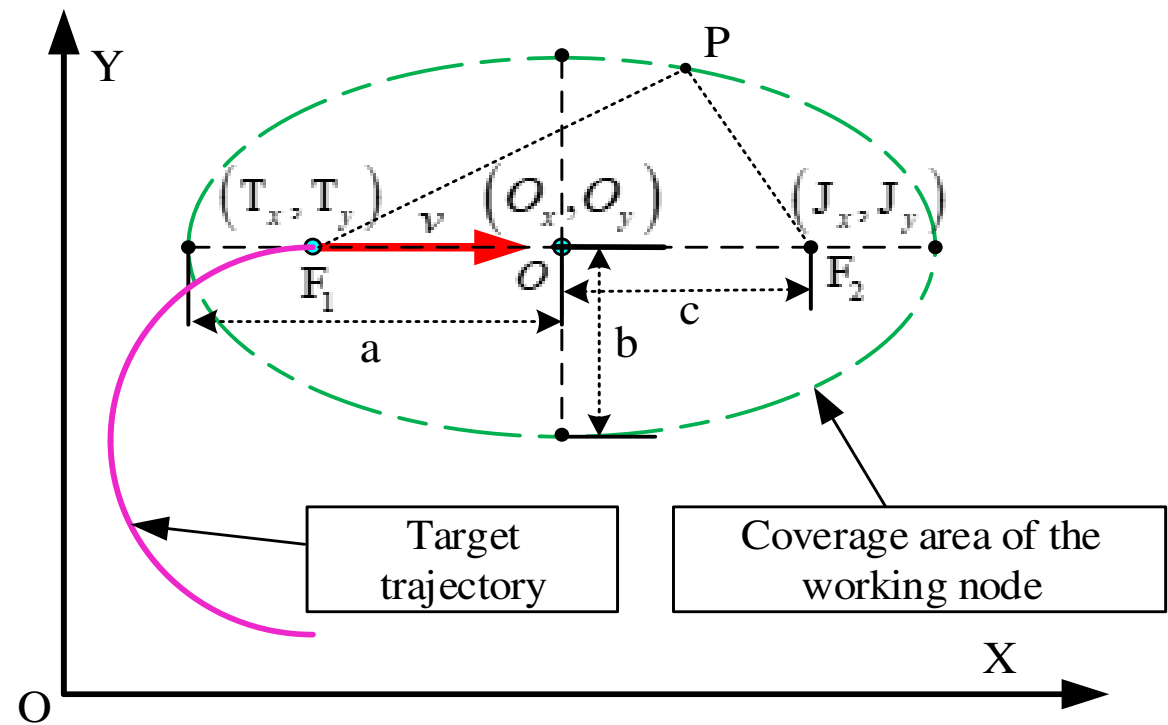

Fig. 2 Target motion trajectory and elliptical area 


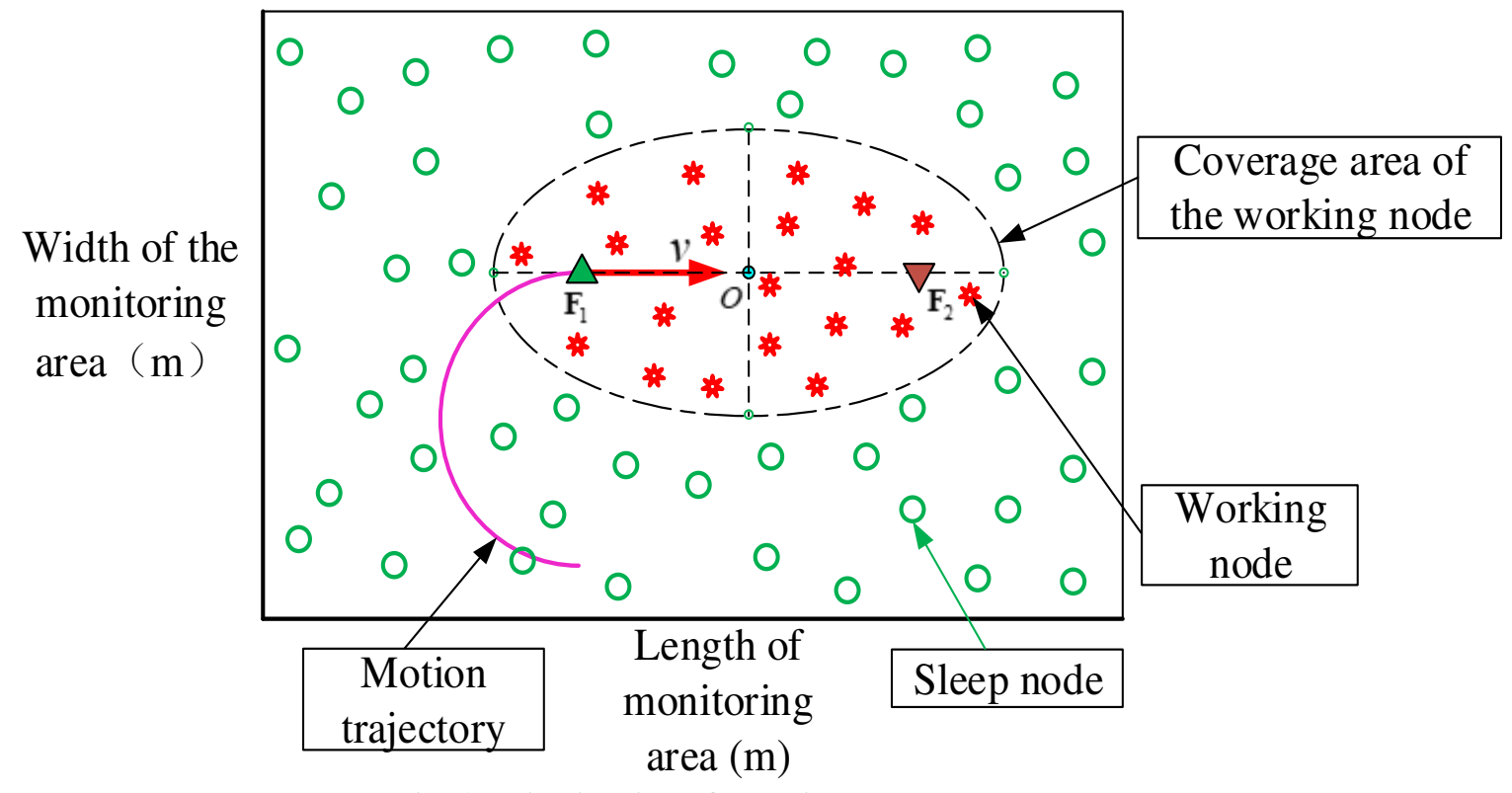

Fig. 3 Distribution of working nodes and sleep nodes

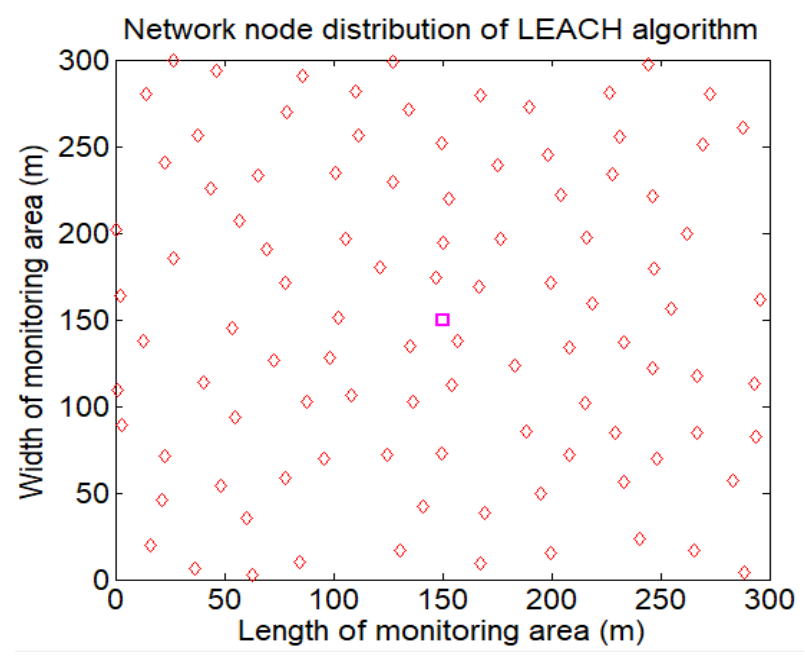

(a)

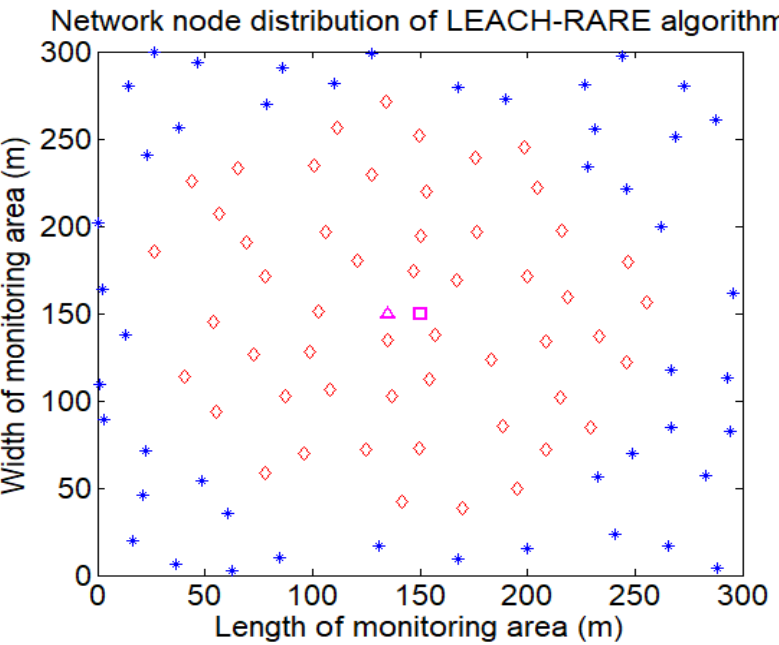

(c)

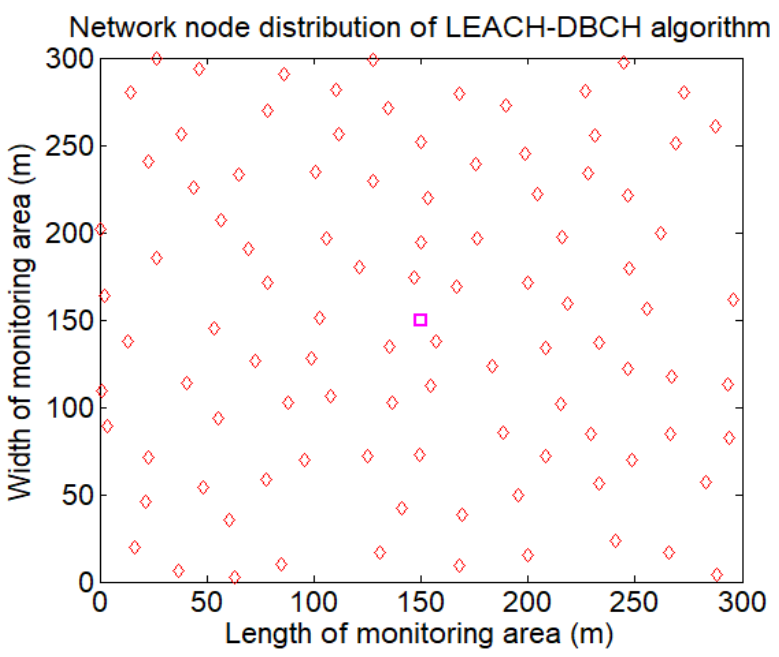

(b)

Network node distribution of LEACH-MTC algorithm

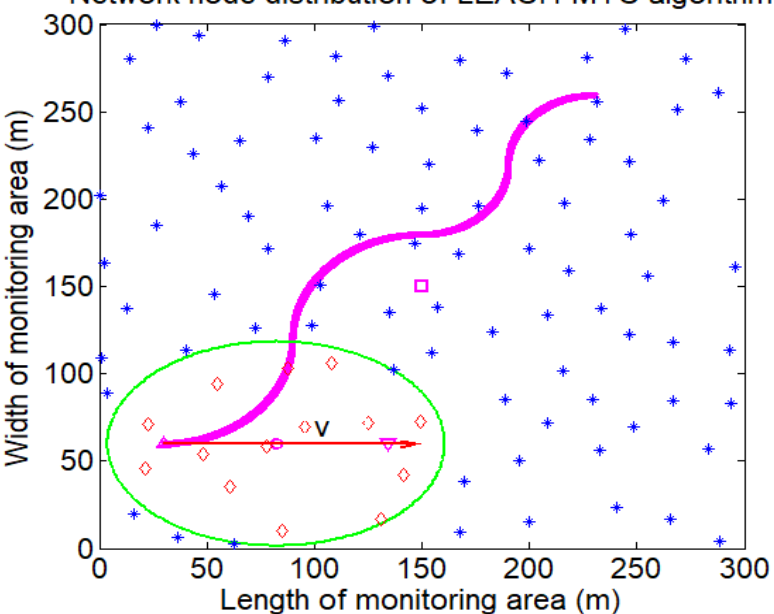

(d)

Fig.4 Node distribution of four algorithms 


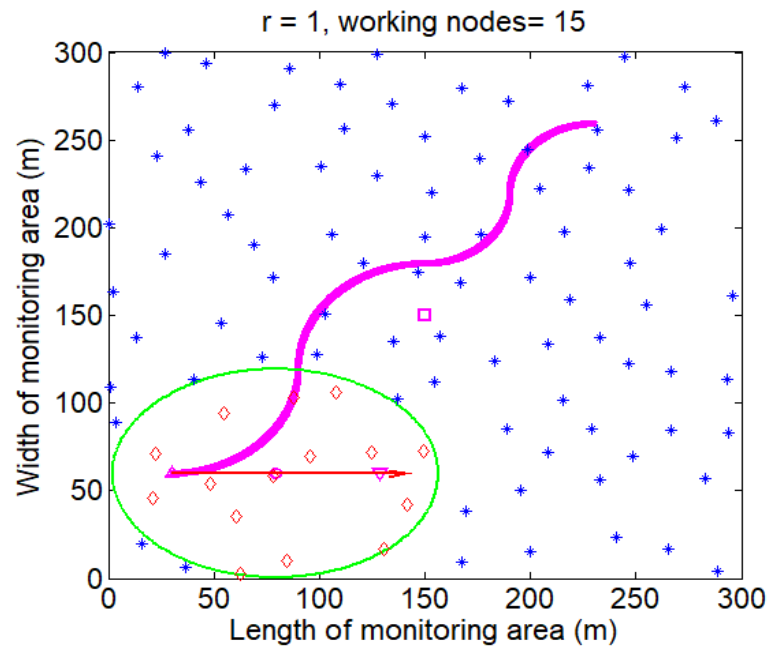

(a)

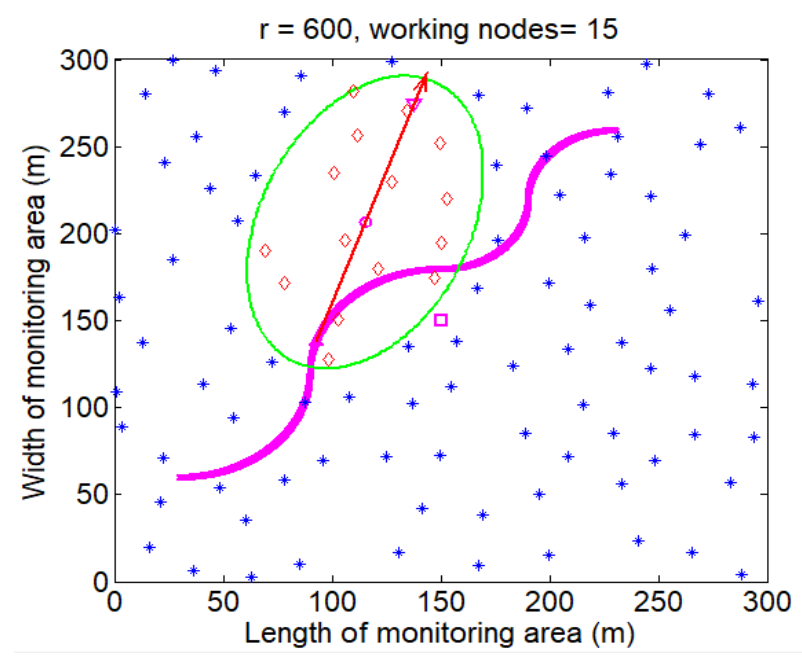

(c)

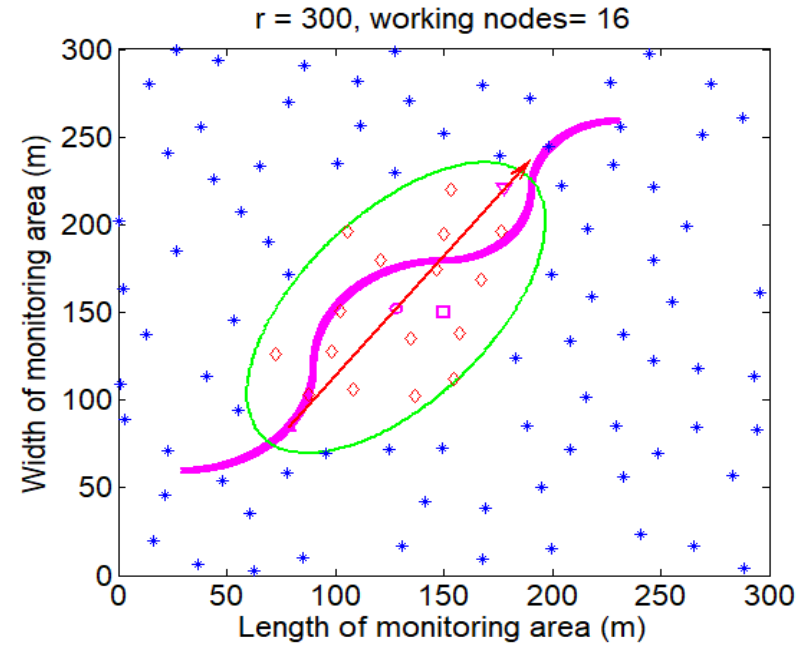

(b)

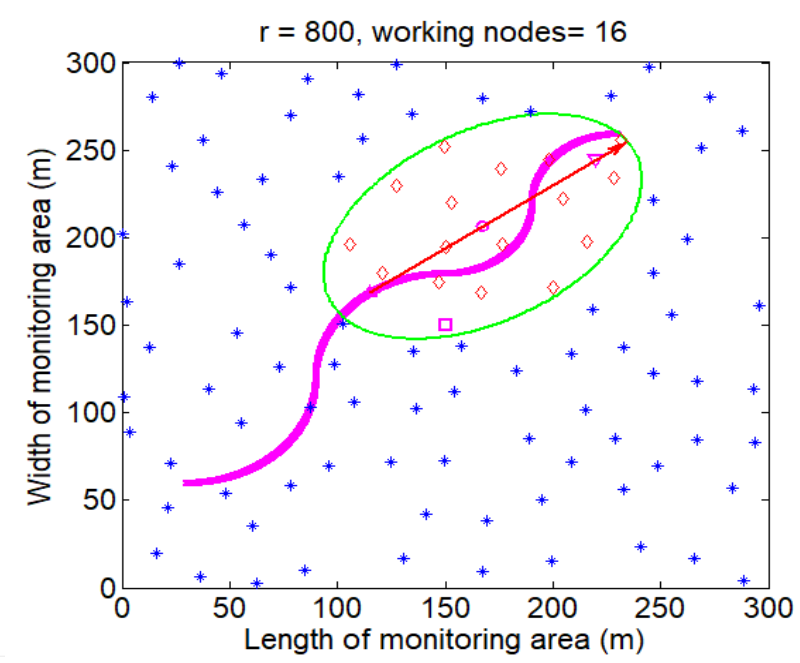

(d)

Fig.5 Change of target position and working node selection

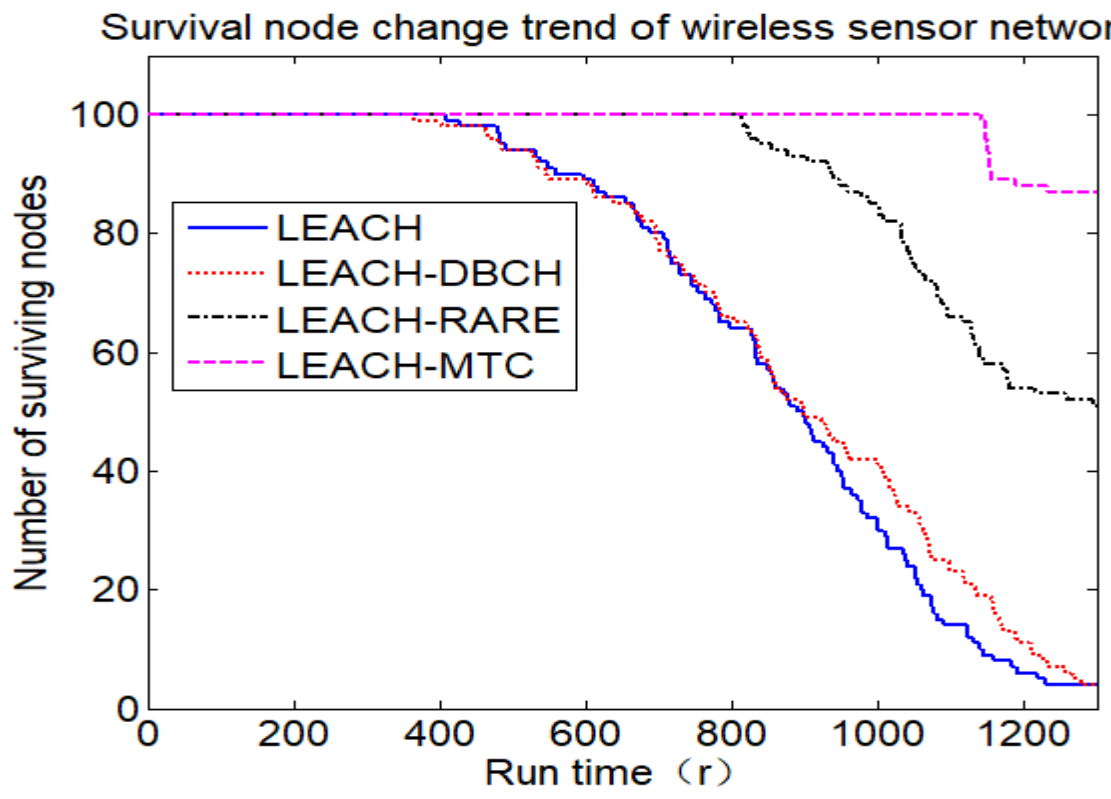

Fig. 6 The number of network surviving nodes 


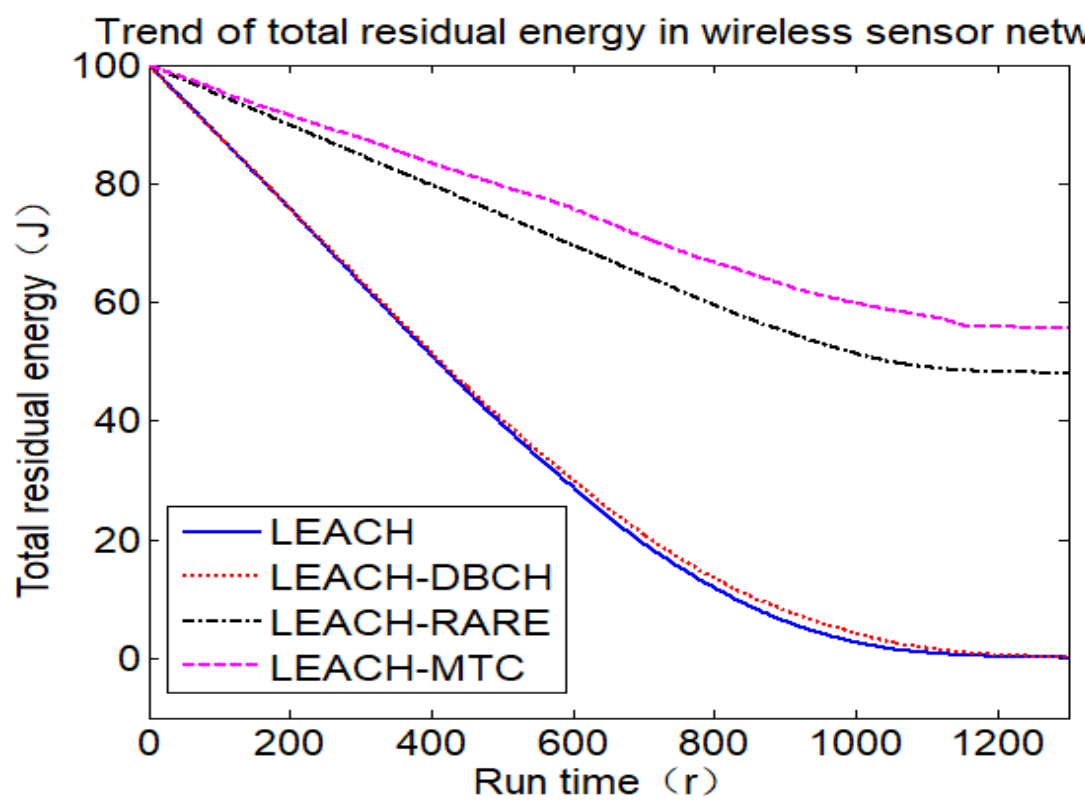

Fig.7 The variation of network residual energy 


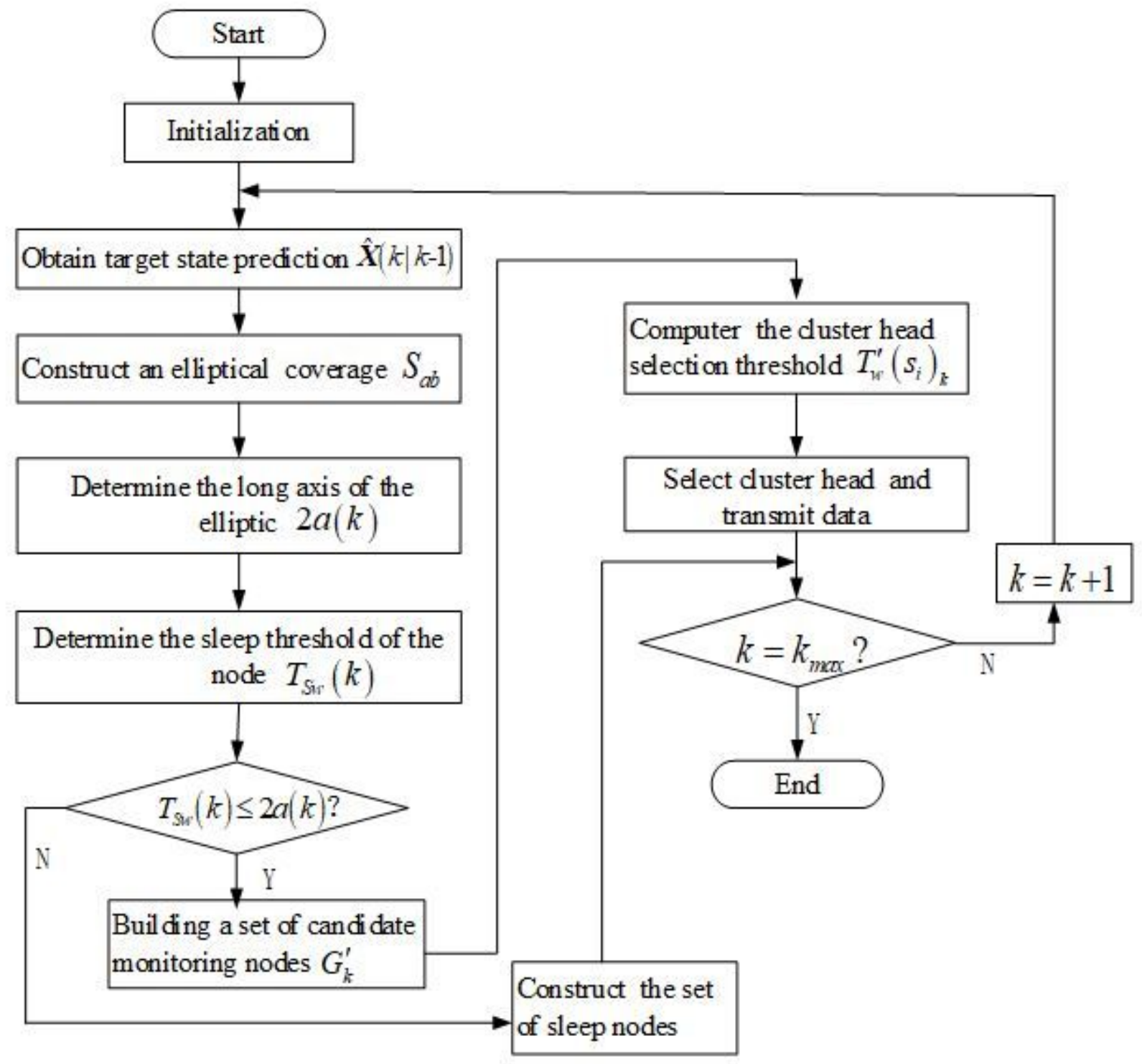

Figure 1

LEACH-MTC algorithm flow chart 


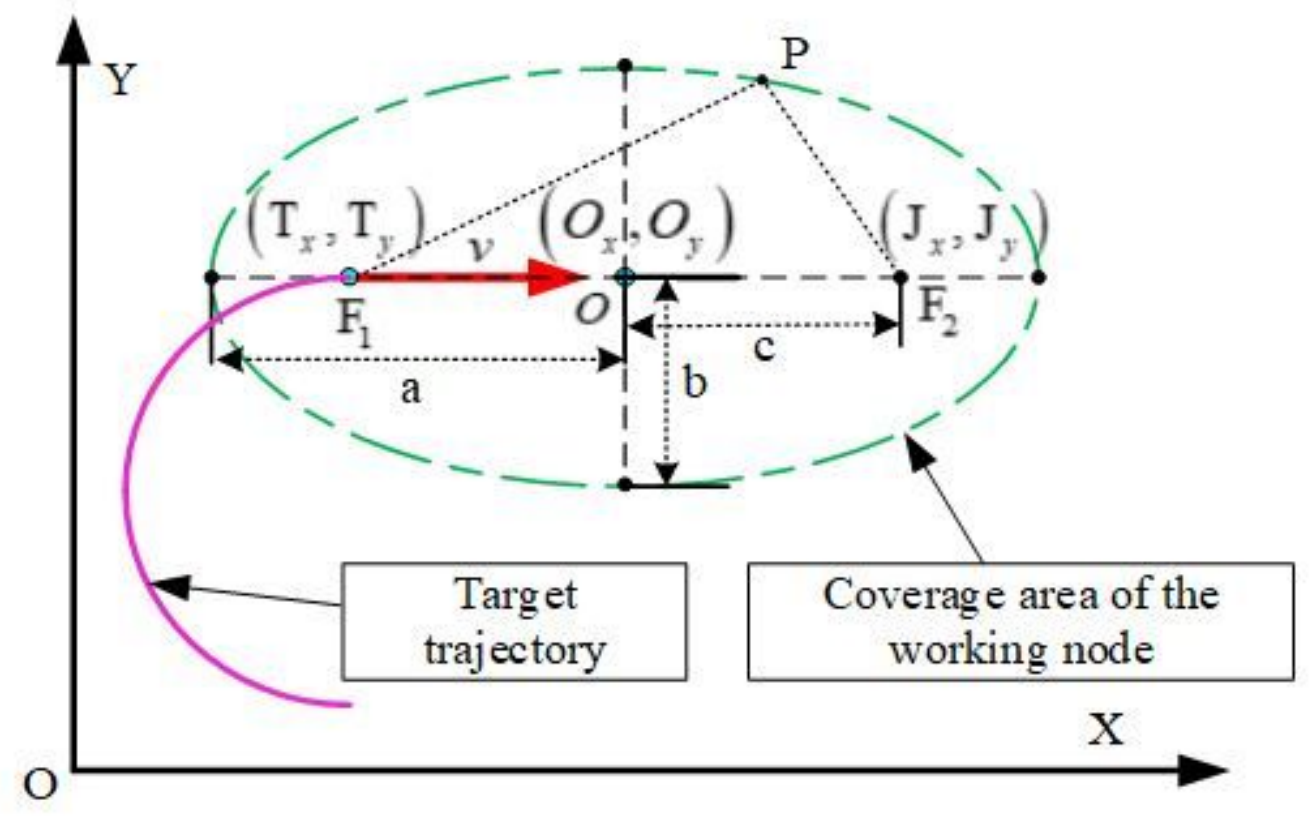

Figure 2

Target motion trajectory and elliptical area

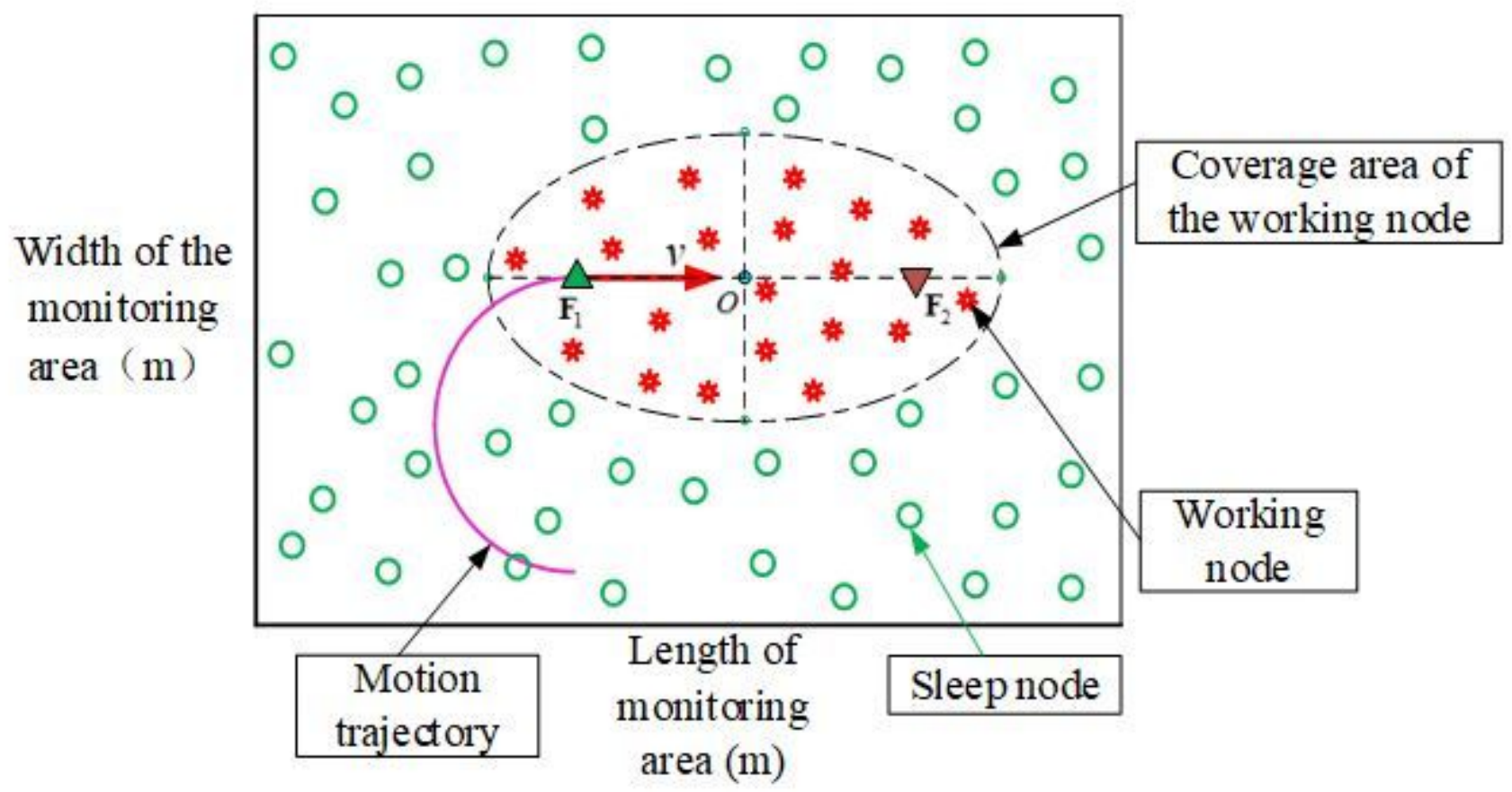

Figure 3 
Distribution of working nodes and sleep nodes

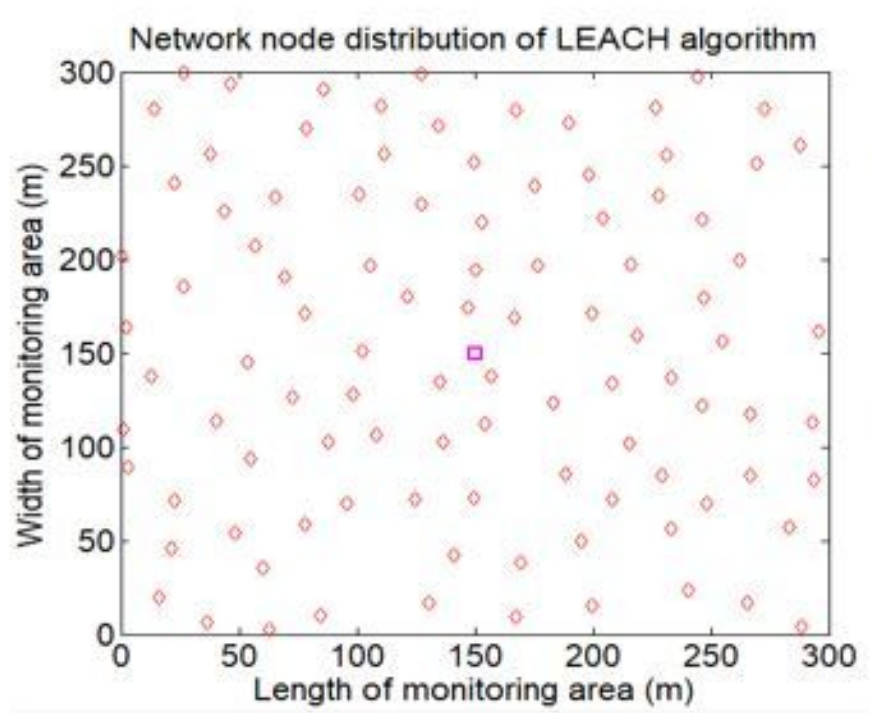

(a)

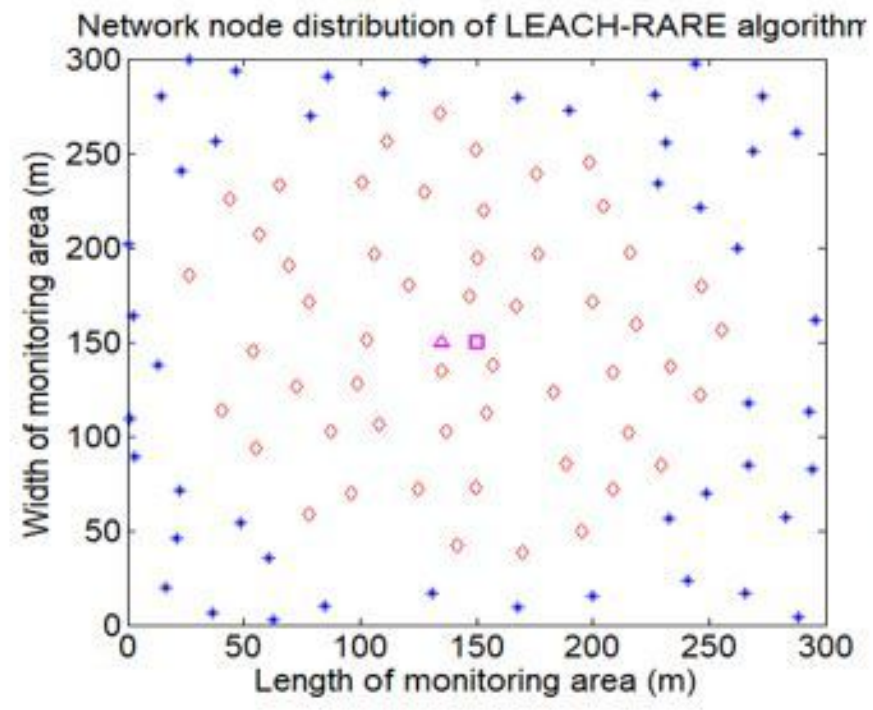

(c)

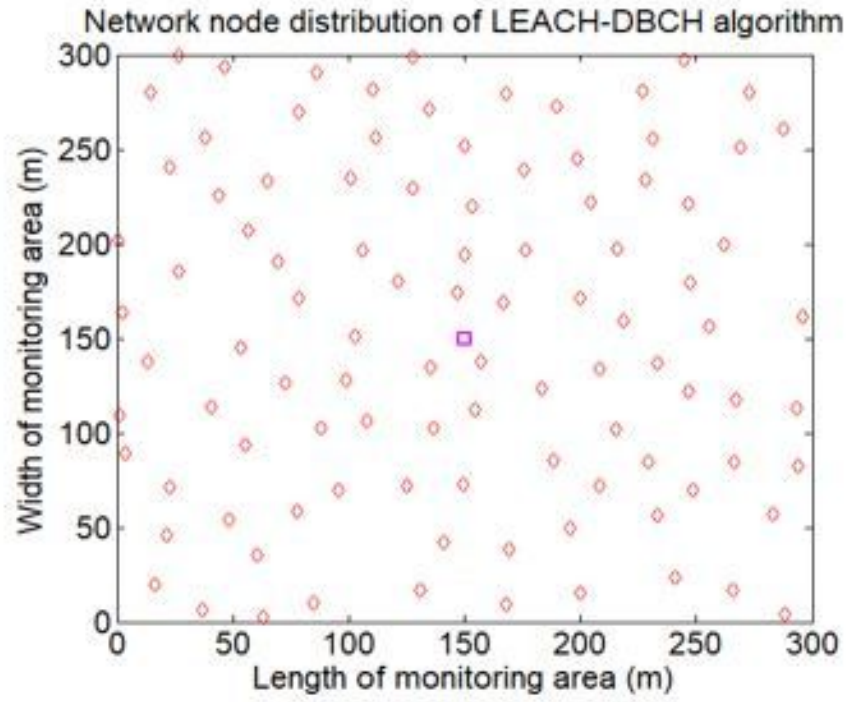

(b)

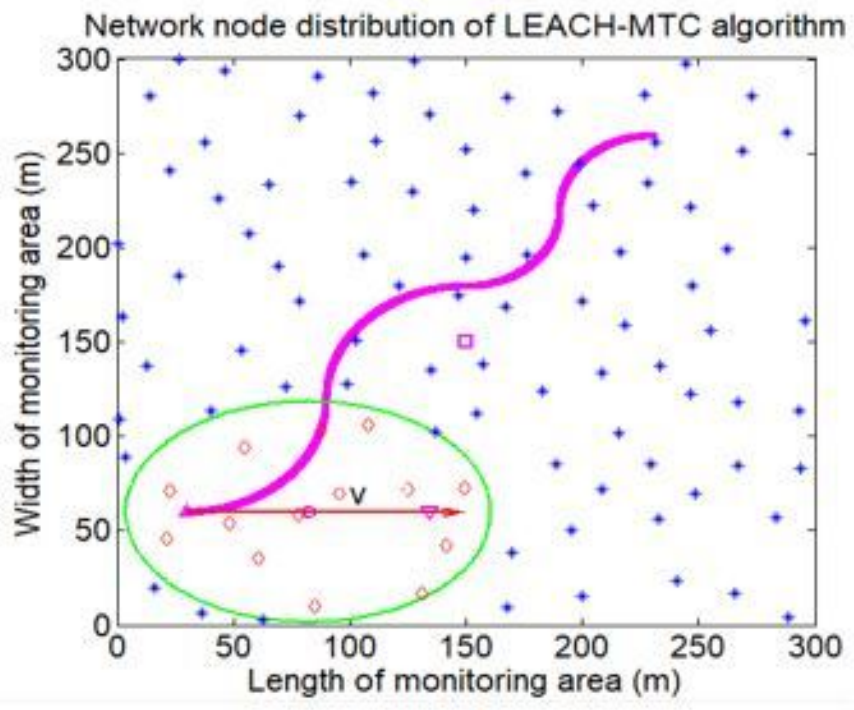

mmno(d)

\section{Figure 4}

Node distribution of four algorithms 


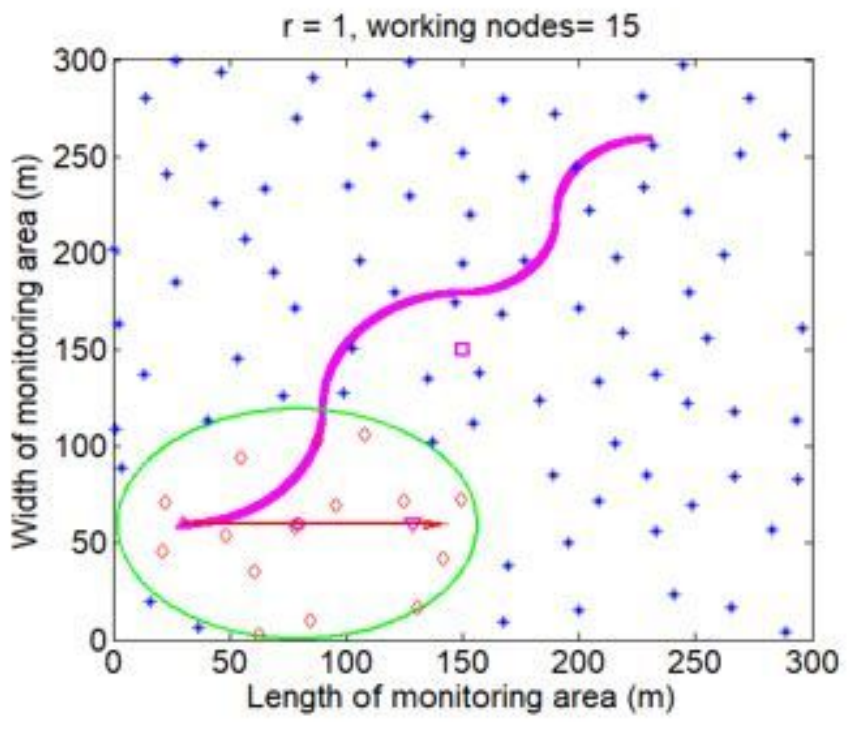

(a)

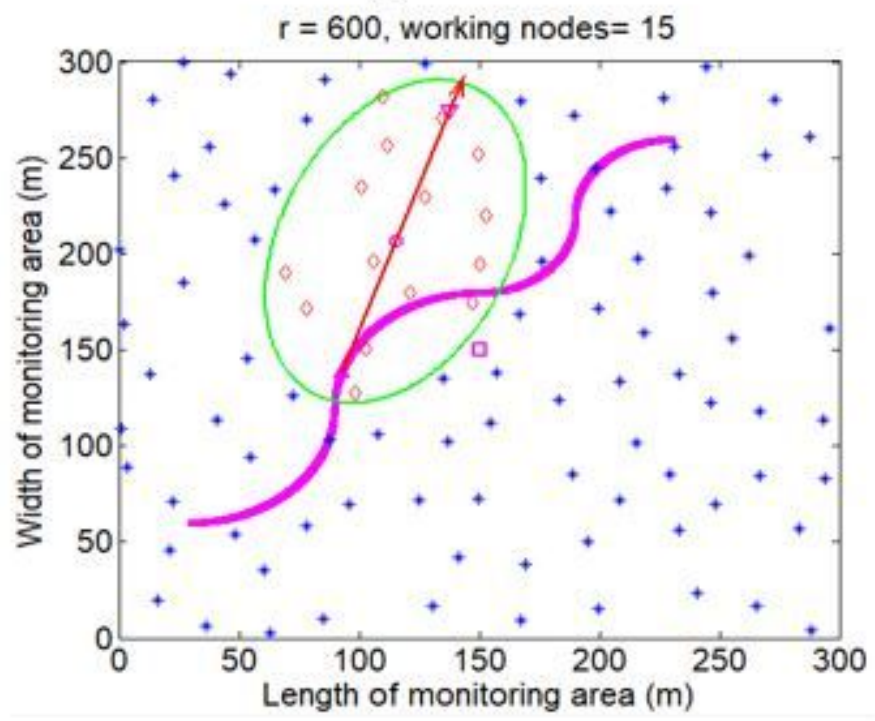

(c)

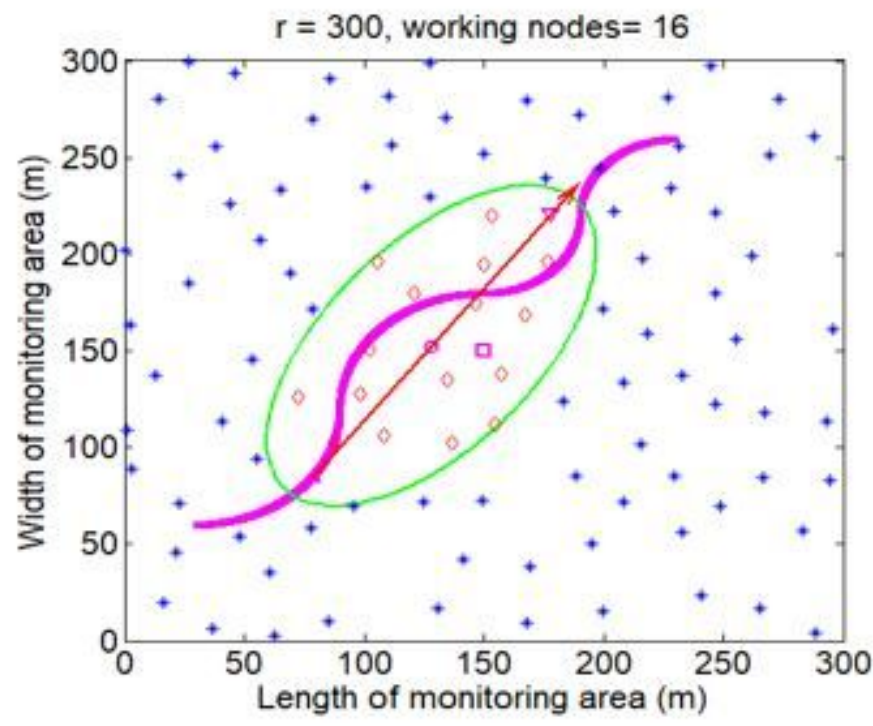

(b)

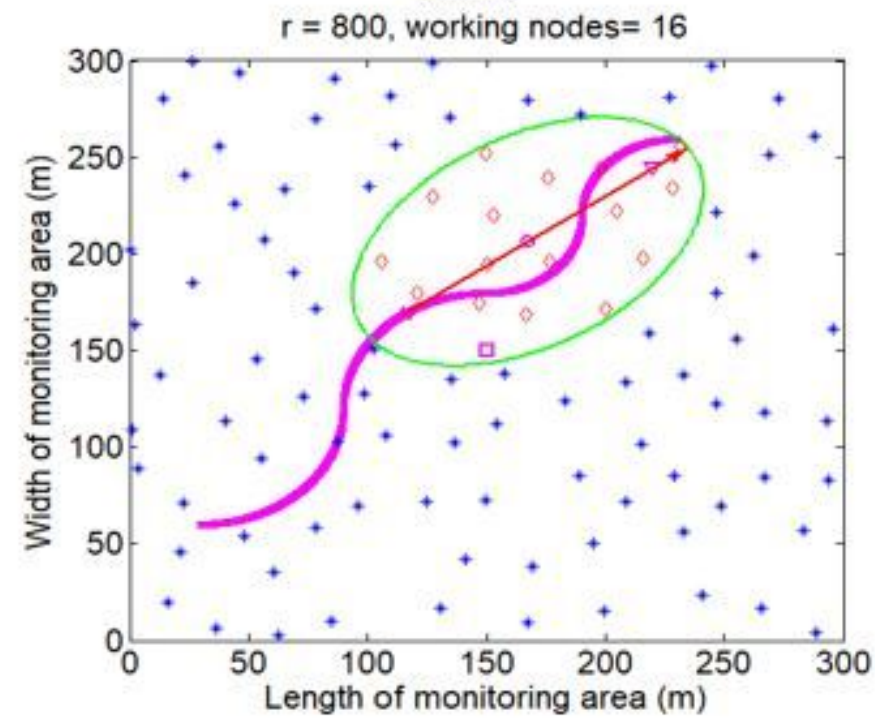

(d)

Figure 5

Change of target position and working node selection 


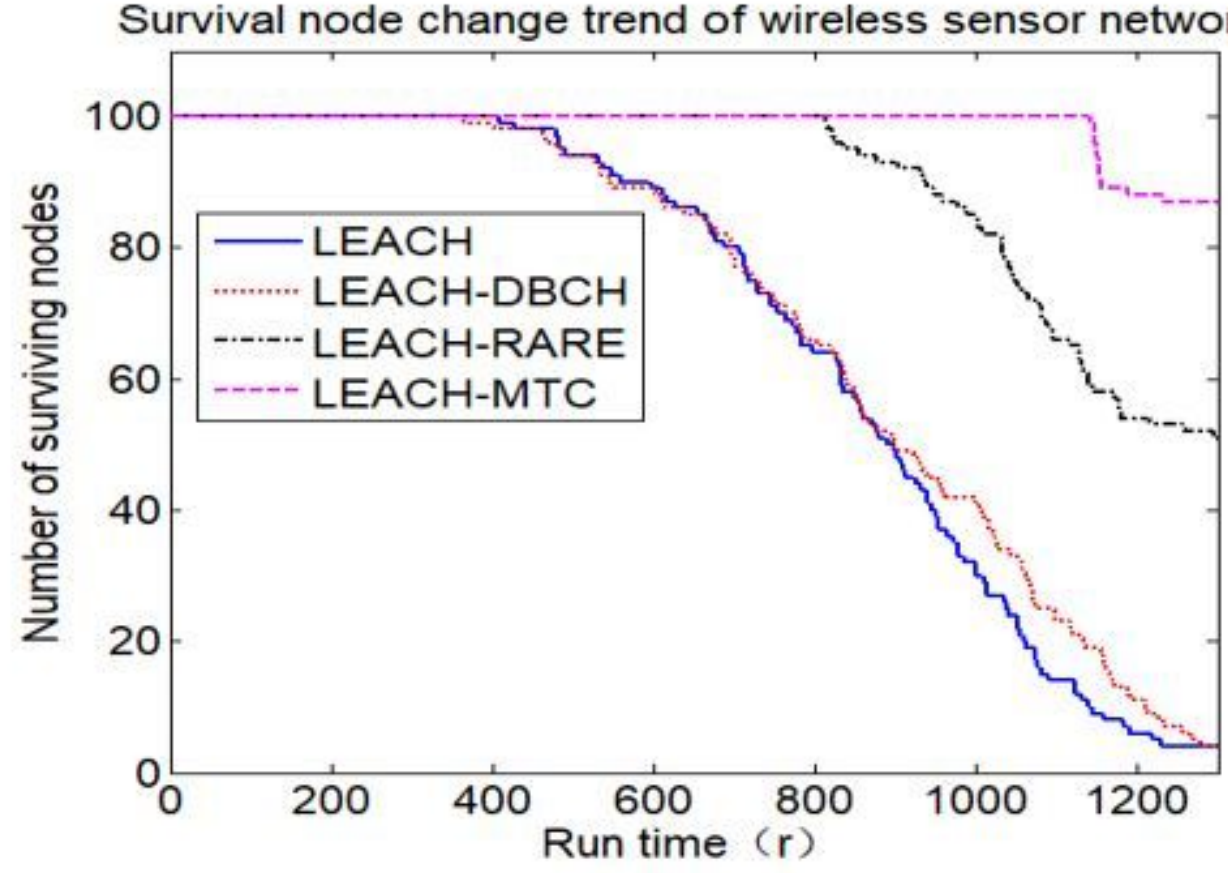

Figure 6

The number of network surviving nodes

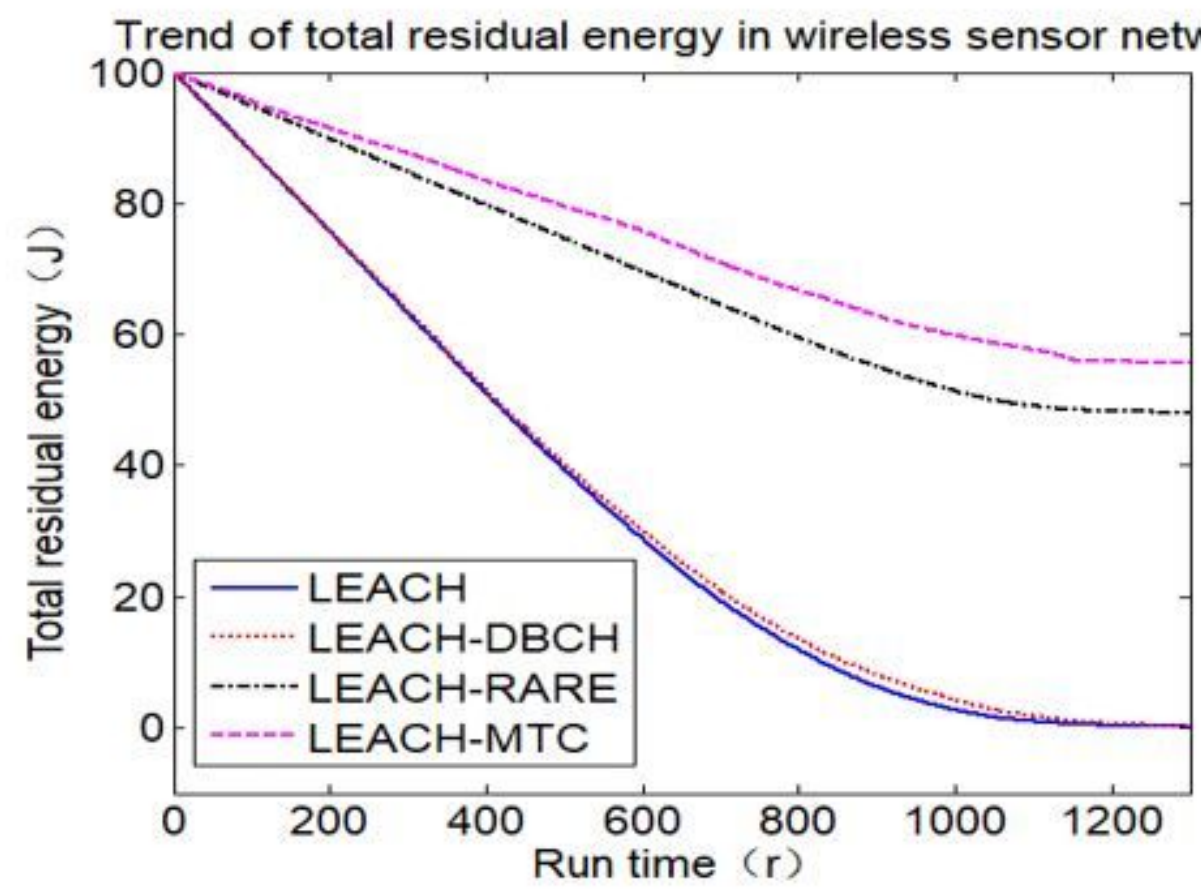

Figure 7 
The variation of network residual energy 